\title{
Distributed Adaptive Fixed-Time Tracking Consensus Control for Multiple Uncertain Nonlinear Strict-Feedback Systems under a Directed Graph
}

\author{
Pinwei Li $\mathbb{D}$, ${ }^{1}$ Jiyang Dai $\mathbb{D}^{1,2}{ }^{1,2}$ Jin Ying $\mathbb{D}^{1},{ }^{1}$ Zhe Zhang $\mathbb{D}^{1},{ }^{1}$ and Cheng He $\mathbb{D}^{1}$ \\ ${ }^{1}$ School of Information Engineering, Nanchang Hangkong University, Nanchang 330063, China \\ ${ }^{2}$ Key Laboratory of Nondestructive Testing, Nanchang Hangkong University, Ministry of Education, Nanchang 330063, China \\ Correspondence should be addressed to Jiyang Dai; djiyang@163.com
}

Received 9 May 2020; Revised 18 July 2020; Accepted 28 July 2020; Published 26 August 2020

Academic Editor: Danilo Comminiello

Copyright (c) 2020 Pinwei Li et al. This is an open access article distributed under the Creative Commons Attribution License, which permits unrestricted use, distribution, and reproduction in any medium, provided the original work is properly cited.

\begin{abstract}
In this brief, we study the distributed adaptive fixed-time tracking consensus control problem for multiple strict-feedback systems with uncertain nonlinearities under a directed graph topology. It is assumed that the leader's output is time varying and has been accessed by only a small fraction of followers in a group. The distributed fixed-time tracking consensus control is proposed to design local consensus controllers in order to guarantee the consensus tracking between the followers and the leader and ensure the error convergence time is independent of the systems' initial state. The function approximation technique using radial basis function neural networks (RBFNNs) is employed to compensate for unknown nonlinear terms induced from the controller design procedure. From the Lyapunov stability theorem and graph theory, it is shown that, by using the proposed fixed-time control strategy, all signals in the closed-loop system and the consensus tracking errors are cooperatively semiglobally uniformly bounded and the errors converge to a neighborhood of the origin within a fixed time. Finally, the effectiveness of the proposed control strategy has been proved by rigorous stability analysis and two simulation examples.
\end{abstract}

\section{Introduction}

In the past decade, the finite-time consensus control of the multiagent has become one of the hot topics, since the finitetime control has a faster convergence rate, higher precision, and more robustness. However, there is a limitation for finite-time control that the convergence time increases accordingly when the initial state of the system is far from the equilibrium point. To overcome this limitation, fixed-time stability control [1] was proposed in 2012 by Polyakov et al.

In 1965, finite-time control was first proposed, and many related research results emerged in the following decades. Due to the different agent dynamics in multiagent systems (MASs), there are various finite-time control developed $[2-7]$. To list a few, in $[8,9]$, by utilizing the principle of homogeneity [10], the authors proposed finite-time consensus control protocols for first-order and second-order MASs, respectively. In [11], power integrator technique was employed to solve the problem of finite-time consensus control for MASs with double integrator dynamics. Furthermore, in [12], by combining the homogeneous domination method with the power integrator method, the authors proposed two classes of finite-time consensus protocols for MASs composed of first-order and secondorder integrator agents. Thereafter, by using the Lyapunovfunction-based method, several finite-time consensus control strategies were proposed for high-order uncertain MASs [13-15]. Even so, there is a limitation for the finite-time consensus control results that the convergence time is seriously dependent on the MASs' initial states. That is to say, once the initial state is far away from the equilibrium point, the convergence time will increase as a result. In practice, it is much more reasonable for a predicable convergence time.

To overcome the limitation of finite-time control, a serial of research results [16-26] on fixed-time consensus control for MASs has been carried out since its setting time can be 
bounded and predefined. Note that most of the fixed-time control results [16-20] were designed for MASs with first order or second order. Only a few results [21-26] solved the fixed-time consensus problems for high-order MASs. On the contrary, strict-feedback or lower triangular systems have been widely regarded as a control target due to the description of various physical systems [27]. In fact, the strictfeedback systems require the recursive and systematic control design procedure since the control input is not matched with system nonlinearities. In [28], the adaptive backstepping design was proposed to satisfy this requirement. Furthermore, in $[29,30]$, the adaptive neural network backstepping controllers were proposed for a class of strictfeedback nonlinear systems. Nevertheless, there is a problem with using backstepping to design a controller for the strictfeedback systems; it can cause "explosion of complexity." To overcome this problem, in [31], a dynamic surface design technique was proposed for strict-feedback systems. Furthermore, in [32], neural network-based adaptive dynamic surface control was proposed. Subsequently, this method was extended to MASs in [33-35], and a distributed adaptive dynamic surface design technique was developed.

Motivated by the above observations, in this paper, a distributed adaptive fixed-time tracking consensus control is proposed for multiple strict-feedback systems with unknown nonlinearities under a directed graph topology. In the design, we employed $\mathrm{RBF}$ neural networks to compensate for unknown nonlinear terms and some functions that are difficult to calculate induced from the controller design procedure. From Lyapunov stability theorem and fixed-time control theory, by using distributed coordinate conversion, virtual controllers and actual controllers were designed. Finally, a novel distributed fixed-time consensus control protocol is designed for the considered strict-feedback nonlinear MASs, which can guarantee that all signals in the total closed-loop systems are bounded and the tracking errors can quickly converge to the neighborhood of the origin within the fixed time. The principal contributions of this paper are as follows. (1) A novel method to solve distributed adaptive fixed-time tracking consensus control for strict-feedback nonlinear MASs has been proposed. Compared with some existing results [16-26], our control strategy, by using adaptive RBF neural network control algorithm, is applicable to high-order MASs with different unknown nonlinear functions and order of dynamics. (2) In order to solve the "complexity explosion" problem caused by the repeated differentiation in the controller design process, it is different from the traditional dynamic surface technique; this paper constructs a smooth function $M(Z)$ derived from Lyapunov stability to compensate the part of $\left(\partial v_{i} / \partial \widehat{\theta}_{i}\right) \widehat{\theta}_{i}$ in the differential of the virtual controller $v_{i}$, while the other part of the differential is approximated by RBF neural networks. This method greatly simplifies the design process of the controller while ensuring Lyapunov stability. (3) In [36-39], their controller designed have a power function similar to $z^{2 k-1}, 0<k<1$. However, incorrect selection of $k$ will lead to singularity. In this paper, a novel fixed-time controller is proposed to solve this problem. (4) RBF neural networks are introduced to approximate the unknown functions $f_{i}(\cdot)$ in MASs to make our designed fixed-time control can suitable more systems with complex dynamics.

The organization for the remaining part of this paper is given below. Section 2 presents preliminaries and problem description. Sections 3 and 4 give the detailed process of distributed fixed-time control protocol and stability analysis, respectively. In Section 5, the proposed control scheme is proved to be effective through a simulation experiment. Finally, conclusions are summarized in Section 6.

\section{Preliminaries and Problem Description}

2.1. Graph Theory. Let $G=(v, \varepsilon)$ be a directed graph with the set of nodes or vertices $v=\{1, \ldots, M\}$ and the set of edges or arcs $\varepsilon \subseteq v \times v$. An edge $(j, i) \in \varepsilon$ means that agent $i$ can obtain information from agent $j$, but agent $j$ cannot obtain agent $i$ 's. The set of neighbors of a node $i$ is $N_{i}=\{j \mid(j, i) \in \varepsilon\}$, which is the set of nodes with edges incoming to node $i$. The weighted adjacency matrix $A=\left[a_{i j}\right] \in R^{M \times M}$ is defined as $a_{i j}>0$ if $(j, i) \in \varepsilon$ and $a_{i j}=0$ otherwise. Self-loop is not allowed, i.e., $a_{i i}=0$. The Laplacian matrix $L$ is defined as $L=D-A \in R^{M \times M}$, where $D=\operatorname{diag}\left[d_{1}, \ldots, d_{M}\right] ; d_{i}=\sum_{j=1, j \neq i}^{M} a_{i j}$ is the diagonal element of the degree matrix $D$.

A directed path from node $i_{1}$ to node $i_{k}$ is a sequence of edges of the form $\left(i_{1}, i_{2}\right),\left(i_{2}, i_{3}\right), \ldots,\left(i_{k-1}, i_{k}\right)$ in a directed graph. A directed tree is a directed graph where every node has exactly one parent except for the root and the root has directed paths to every other node. A directed graph has a directed spanning tree if there exists at least a node having a directed path to all the other nodes.

2.2. RBFNNs. An RBF neural network [40] is applied in this paper to approximate arbitrary continuous functions. The RBF neural network is defined as follows:

$$
\widehat{f}(Z)=W^{T} S(Z),
$$

where $W=\left[w_{1}, \ldots, w_{l}\right]^{T} \in R^{l}$ is the weight vector, $l>0$ is the number of nodes of the neural network, $Z \in \Omega_{Z} \subset R^{q}$ is the input of the RBF neural network, $q$ is the input dimension, $S(Z)=\left[s_{1}[Z], \ldots, s_{l}(Z)\right]^{T} \in R^{l}$ is the basis vector function, and $s_{i}(Z)$ is the output of the $i$ th neural node. A Gaussian function is always chosen as $s_{i}(Z)$, i.e., $s_{i}(Z)=\exp \left[-\left(Z-\xi_{i}\right)^{T}\left(\left(Z-\xi_{i}\right) / r_{i}^{2}\right)\right], i=1, \ldots, l$, where $r_{i}$ is the width of the base function and $\xi_{i}=\left[\xi_{i 1}, \ldots, \xi_{i q}\right]^{T}$ is the center of the basis function. With a sufficient number $l$ of neural nodes selected, an RBF neural network can approximate arbitrary continuous function $f(Z)$ in a compact set $\Omega_{Z} \in R^{q}$ with arbitrary accuracy $\varepsilon$ :

$$
f(Z)=W^{* T} S(Z)+\delta(Z), \quad \forall Z \in \Omega_{Z} \in R^{q},
$$

where $\delta(Z)$ is the approximation error with $|\delta(Z)| \leq \varepsilon$ and $W^{*}$ is the given ideal constant weight vector, which is defined as follows: 


$$
W^{*}=\arg \min _{W \in R^{l}} \sup _{Z \in \Omega_{Z}}\{|f(Z)-\hat{f}(Z)|\} .
$$

In this paper, let $\theta=\max \left\{\left(\left\|W^{*}\right\|\right) / b\right\}$ with $\tilde{\theta}=\theta-\widehat{\theta}$, where $\hat{\theta}$ are the estimates of the unknown constants $\theta, W^{*}$ is the ideal weight vector of the RBF neural network, $b$ is a positive design parameter, and $\|\cdot\|$ is the norm.

Lemma 1 (see [41]). Consider the Gaussian function (1); $\|S(Z)\|$ has an upper bound such that

$$
\|S(Z)\| \leq \sum_{k=0}^{\infty} 3 q(k+2)^{q-1} e^{-\left(\left(2 p^{2} k^{2}\right) / r^{2}\right)}:=s,
$$

where $p=(1 / 2)\left(\min _{i \neq j}\left\|\xi_{i}-\xi_{j}\right\|\right)$.

Remark 1. Lemma 1 has been proved in [41, 42]. Since $\sum_{k=0}^{\infty} 3 q(k+2)^{q-1} e^{-\left(\left(2 p^{2} k^{2}\right) / r^{2}\right)}$ is convergent, $s$ is a limited value. In addition, $s$ is independent of the neural network node numbers $l$ and the neural network inputs $Z$.

Assumption 1 (see [36]). There are unknown constants $Q_{i}$ that make $\left|\widetilde{\theta}_{i}\right| \leq Q_{i}<\infty, i=1,2, \ldots, n$.

\subsection{Fixed Time}

Definition 1. Consider the following nonlinear system:

$$
\begin{aligned}
\dot{x}(t) & =f(t, x), \\
x(0) & =x_{0},
\end{aligned}
$$

where $x \in R^{n}$ and $f: R_{+} \times R^{n} \longrightarrow R^{n}$, and assume that the origin is an equilibrium point.

Lemma 2 (see [22]). If there exist design constants $\phi_{1}>0$, $\phi_{2}>0, \alpha \in(0,1)$, and $\beta \in(1,+\infty)$ such that

$$
\dot{V} \leq-\phi_{1} V^{\alpha}(x)-\phi_{2} V^{\beta}(x),
$$

where $V(x)$ is a continuous differentiable positive definite function; system (5) is global fixed-time stable, and the fixed convergence time satisfies

$$
T \leq T_{\max }:=\frac{1}{\phi_{1}(1-\alpha)}+\frac{1}{\phi_{2}(\beta-1)} .
$$

Remark 2. The sufficient conditions and convergence time for finite-time and fixed-time consensus control schemes are shown in Table 1. Where $\mu_{1}, \mu_{2}, \rho_{1}$, and $\rho_{2}$ are positive design parameters, $\alpha>1$, and $0<\beta<1$. From convergence time, it can be seen that the finite-time control is related to the initial state $x(0)$, while the fixed-time control is not.

Lemma 3 (see [36]). If there exist design constants $\phi_{1}>0$, $\phi_{2}>0, \alpha \in(0,1), \beta \in(1,+\infty), \tau \in(0, \infty)$, and $\varpi \in(0,1)$ such that

$$
\dot{V} \leq-\phi_{1} V^{\alpha}(x)-\phi_{2} V^{\beta}(x)+\tau .
$$

Then, the origin of system (5) is practical fixed-time stable and the fixed-time Tcan be estimated by

$$
T \leq T_{\max }:=\frac{1}{\phi_{1} \bowtie(1-\alpha)}+\frac{1}{\phi_{2} \bowtie(\beta-1)} .
$$

The residual set of the solution of system (5) is given by

$$
x \in\left\{V(x) \leq \min \left\{\left(\frac{\tau}{(1-\varpi) \phi_{2}}\right)^{1 / \beta},\left(\frac{\tau}{(1-\varpi) \phi_{1}}\right)^{1 / \alpha}\right\}\right\} \text {. }
$$

Lemma 4 (see [43]). Let $x_{1}, x_{2}, \ldots, x_{n} \geq 0$. Then,

$$
\begin{aligned}
& \sum_{i=1}^{n} x_{i}^{\rho} \geq\left(\sum_{i=1}^{n} x_{i}\right)^{\rho}, \quad \text { if } 0<\rho \leq 1, \\
& \sum_{i=1}^{n} x_{i}^{\rho} \geq n^{1-\rho}\left(\sum_{i=1}^{n} x_{i}\right)^{\rho}, \quad \text { if } 1<\rho<\infty .
\end{aligned}
$$

Lemma 5 (see [44]). For any variable $x \in R$ and any positive constant $\kappa$, the following relationship holds:

$$
0 \leq|x|<\kappa+\frac{x^{2}}{\sqrt{x^{2}+\kappa^{2}}} .
$$

Lemma 6 For $y \geq x>0, x, y \in R$ and any positive constant $\delta$, then satisfying

$$
\frac{y}{\sqrt{y+\delta}} \geq \frac{x}{\sqrt{x+\delta}}
$$

Proof

$$
\begin{gathered}
\frac{y}{\sqrt{y+\delta}}-\frac{x}{\sqrt{x+\delta}}=\frac{y+\delta}{\sqrt{y+\delta}}-\frac{x+\delta}{\sqrt{x+\delta}}-\left(\frac{\delta}{\sqrt{y+\delta}}-\frac{\delta}{\sqrt{x+\delta}}\right) \\
=(\sqrt{y+\delta}-\sqrt{x+\delta})+\left(\frac{\delta}{\sqrt{x+\delta}}-\frac{\delta}{\sqrt{y+\delta}}\right) \geq 0 .
\end{gathered}
$$

2.4. Problem Statement. Suppose that there exist one leader agent, labeled as 0 , and $M$ follower agents, labeled as 1 to $M$, under a directed communication graph topology. The dynamic models of $M$ followers in the strict-feedback form are considered as follows:

$$
\left\{\begin{array}{l}
\dot{x}_{i, k}=x_{i, k+1}+f_{i, k}\left(\bar{x}_{i, k}\right), \\
\dot{x}_{i, n_{i}}=u_{i}+f_{i, n_{i}}\left(x_{i}\right) \\
y_{i}=x_{i, 1}
\end{array}\right.
$$

where $i=1, \ldots, M, k=1, \ldots, n_{i}-1, \bar{x}_{i, k}=\left[x_{j, 1}^{T}, \ldots, x_{i, k}^{T}\right]^{T}$; $x_{i, l} \in R^{p}$ with $l=1, \ldots, k, x_{i}=\left[x_{i, 1}^{T}, \ldots, x_{i, n_{i}}^{T}\right]^{1} \in R^{p n_{i}}$, and $u_{i} \in R^{p}$ are the state vector and the control input of the $i$ th follower, respectively, and $y_{i} \in R^{p}$ is the output of the $i$ th 
TABle 1: The sufficient condition and convergence time for finite-time control and fixed-time control.

\begin{tabular}{lcc}
\hline Control algorithm & Sufficient condition & Convergence time \\
\hline Finite-time control & $\dot{V}(x) \leq-\mu_{2} V(x)^{\beta}$ & $T \leq T_{\max }:=\left(1 / \rho_{2}(1-\beta)\right) V(x(0))^{1-\beta}$ \\
Fixed-time control & $\dot{V}(x) \leq-\mu_{1} V(x)^{\alpha}-\mu_{2} V(x)^{\beta}$ & $T \leq T_{\max }:=\left(1 / \rho_{1}(\alpha-1)\right)+\left(1 / \rho_{2}(1-\beta)\right)$ \\
\hline
\end{tabular}

follower. $f_{i, k}\left(\bar{x}_{i, k}\right) \in R^{p}$ Are $C^{1}$ nonlinear function vectors. It is assumed that the leader's motion is independent of the followers' motion.

The communication topology for the $M+1$ agents is described by a directed graph $G=(v, \varepsilon)$ with $v=\{0,1, \ldots, M\}$, To represent the communications among followers, we define a subgraph as $\bar{G}=(\bar{v}, \bar{\varepsilon})$ with $\bar{v}=\{1, \ldots, M\}$. The adjacency matrix $\bar{A}$ of the subgraph $\bar{G}$ is $\bar{A}=\left[a_{i j}\right] \in R^{M \times M} ; a_{i j}>0$ if $(j, i) \in \bar{\varepsilon}, a_{i j}=0$ otherwise, and $a_{i i}=0$. Then, the Laplacian matrix $L$ is defined as

$$
L=\left[\begin{array}{cc}
0 & 0_{1 \times M} \\
-h & \bar{L}+H
\end{array}\right]
$$

where $h=\left[h_{1}, \ldots, h_{M}\right]^{T}$, with $h_{i}>0$ if the leader $0 \in N_{i}$ and $h_{i}=0$ otherwise, which denotes the communication weight from the leader to followers, $H=\operatorname{diag}\left[h_{1}, \ldots, h_{M}\right]$, and $\bar{L}=$ $\bar{D}-\bar{A}$ with $\bar{D}=\operatorname{diag}\left[d_{1}, \ldots, d_{M}\right]$ is the Laplacian matrix of the subgraph denoting the communication among followers.

Remark 3. For the simplicity of analysis, in the following, we only consider the case where $p=1$. The analysis and main results still hold for any dimension $p$ by using the Kronecker product.

Remark 4. If the directed graph $G$ has a spanning tree, $\operatorname{rank}(L)=M$ [45]. Then, $\operatorname{rank}(\bar{L}+H)=M$ from $(\bar{L}+H) 1_{M}=h$, where $1_{M}$ is an $M$-vector of all ones. Therefore, $\bar{L}+H$ is invertible.

Assumption 2. The $C^{1}$ nonlinear functions $f_{i, k}$ are unknown on a directed graph $G$.

Assumption 3. The leader output signal $r(t) \in R^{p}$ is an $n_{i}$-order differentiable and bounded function and available for the $i$ th followers satisfying $0 \in N_{i}, i=1, \ldots, M$.

Definition 2 (see [33]). The distributed consensus tracking errors for nonlinear followers (15) under the communication graph are said to be cooperatively semiglobally uniformly ultimately bounded (CSUUB) if there exist adjustable constants $c_{1}>0$ and $c_{2}>0$, and the bounds $\beta_{1}>0$ and $\beta_{2}>0$, independent of $t_{0}$, and for every $\alpha_{1} \in\left(0, c_{1}\right)$ and $\alpha_{2} \in\left(0, c_{2}\right)$, there is a time $T \geq 0$, independent of $t_{0}$, such that $\| y_{i}\left(t_{0}\right)-$ $r\left(t_{0}\right)\left\|\leq \alpha_{1} \Rightarrow\right\| y_{i}(t)-r(t) \| \leq \beta_{1}$ and $\left\|y_{i}\left(t_{0}\right)-y_{j}\left(t_{0}\right)\right\| \leq \alpha_{2} \Rightarrow$ $\left\|y_{i}(t)-y_{j}(t)\right\| \leq \beta_{2}$ for all $t \geq t_{0}+T$, where $i=1, \ldots, M$, $j=1, \ldots, M, i \neq j$ and $k=1, \ldots, n$.

The objective of this brief is to design RBF-neural networks-based distributed fixed-time consensus control laws $u_{i}$ for $M$ followers (15) with unknown nonlinearities so that, under the directed graph, the follower outputs $y_{i}$ synchronize to the dynamic leader output $r$ within fixed time while all signals in the total closed-loop systems are bounded.

Remark 5. The strict-feedback system (15) can describe many state-space models of nonlinear systems, i.e., various physical systems, such as flight systems, biochemical process, jet engine, and robotic systems [28]. Therefore, system (15) under a graph topology can represent multiagent systems consisting of several practical applications with different dynamics.

Remark 6. Notice that followers (15) can have various forms. That is, a group of the followers with different nonlinear functions and order of the dynamics can be considered in this brief.

Remark 7. Compared with the previous consensus works, this brief considers the consensus problem of a group of agents consisting of nonlinear followers with nonlinearities unmatched in the control input. Besides, the nonlinearities are unknown under the total communication topology, and the tracking convergence time independent of the initial state of system (15).

\section{Distributed Fixed-Time Consensus Controller Design}

The design procedure on the $i$ th follower contains $n_{i}$ steps. The distributed backstepping design coordinate transformation is as follows:

$$
\begin{aligned}
& z_{i, 1}=\sum_{j=1}^{M} a_{i j}\left(y_{i}-y_{j}\right)+h_{i}\left(y_{i}-y_{0}\right), \\
& z_{i, k}=x_{i, k}-v_{i, k-1},
\end{aligned}
$$

where $i=1, \ldots, M$ and $k=2, \ldots, n_{i}$ and $v_{i, k}$ is the virtual controller of the $k$ th subsystem of $i$ thfollower.

An RBF neural network is used in this paper to approximate the unknown functions $\widehat{f}_{i . k}\left(Z_{i, k}\right)$ :

$$
\hat{f}_{i . k}\left(Z_{i, k}\right)=W_{i, k}^{* T} S_{i, k}\left(Z_{i, k}\right)+\delta_{i, k}\left(Z_{i, k}\right),
$$

and the inequalities involved in the following text are as below:

$$
\begin{gathered}
z_{i, k} \delta_{i, k}\left(Z_{i, k}\right) \leq b_{i} \varsigma_{i, k_{3}} z_{i, k}^{2}+\frac{\varepsilon_{i, k}^{2}}{4 b_{i} \varsigma_{i, k_{3}}} \\
z_{i, k} W_{i, k}^{* T} S_{i, k}\left(Z_{i, k}\right) \leq \frac{b_{i} \theta_{i, k}}{2 \eta_{i, k}^{2}} S_{i, k}^{T}\left(Z_{i, k}\right) S_{i, k}\left(Z_{i, k}\right) z_{i, k}^{2}+\frac{\eta_{i, k}^{2}}{2},
\end{gathered}
$$




$$
\begin{aligned}
& -b_{i} \varsigma_{i, k_{2}} 2^{(1+\beta) / 2}\left(\left(\frac{1}{2} z_{i, k}^{2}\right)^{(1+\beta) / 2}+\left(\frac{b_{i} \widetilde{\theta}_{i, k}^{2}}{2 \gamma_{i}}\right)^{(1+\beta) / 2}\right) \\
& \leq-2 b_{i} \varsigma_{i, k_{2}}\left(\frac{1}{2} z_{i, k}^{2}+\frac{b_{i} \widetilde{\theta}_{i, k}^{2}}{2 \gamma_{i}}\right)^{(1+\beta) / 2}, \\
& -b_{i} \varsigma_{i, k_{1}} 2^{(1+\alpha) / 2}\left(\left(\frac{1}{2} z_{i, k}^{2}\right)^{(1+\alpha) / 2}+\left(\frac{b_{i} \widetilde{\theta}_{i, k}^{2}}{2 \gamma_{i}}\right)^{(1+\alpha) / 2}\right) \\
& \leq-2 b_{i} \varsigma_{i, k_{1}}\left(\frac{1}{2} z_{i, k}^{2}+\frac{b_{i} \widetilde{\theta}_{i, k}^{2}}{2 \gamma_{i}}\right)^{(1+\alpha) / 2},
\end{aligned}
$$

where $Z_{i, k}=\left[x_{i, 1}, \ldots, x_{i, k}, \hat{\theta}_{i, 1}, \ldots, \hat{\theta}_{i, k}, h_{i} y_{0}, h_{i} \dot{y}_{0}, \ldots, h_{i} y_{0}\right.$ (k), $\left.a_{i 1} y_{1,1}, \ldots, a_{i M} y_{M, 1}, \ldots, a_{i 1} y_{1, k}, \ldots, a_{i M} y_{M, k}\right] \quad$ with $i=1, \ldots, M, k=1, \ldots, n_{i}$ is the input vector, and $b_{i}, \varsigma_{i, k_{3}}$, $\varsigma_{i, k_{2}}, \varsigma_{i, k_{1}}, \varepsilon_{i, k}, \eta_{i, k}$, and $\gamma_{i}$ are positive design parameters.

Step 1. according to (15), (17), and (18), we have

$$
\dot{z}_{i, 1}=\left(d_{i}+h_{i}\right) z_{i, 2}+\left(d_{i}+h_{i}\right) v_{i, 1}+\hat{f}_{i, 1}\left(Z_{i, 1}\right)-\sum_{j=1}^{M} a_{i j} x_{j, 2} \text {, }
$$

where $\hat{f}_{i, 1}\left(Z_{i, 1}\right)=\left(d_{i}+h_{i}\right) f_{i, 1}-\sum_{j=1}^{M} a_{i j} f_{j, 1}-h_{i} \dot{y}_{0}$.

Construct a Lyapunov function as

$$
V_{i, 1}=\frac{1}{2} z_{i, 1}^{2}+\frac{b_{i} \widetilde{\theta}_{i, 1}^{2}}{2 \gamma_{i}}
$$

Time differentiation of $V_{i}$ yields

$$
\dot{V}_{i, 1}=\left(d_{i}+h_{i}\right) z_{i, 1} z_{i, 2}+\left(d_{i}+h_{i}\right) z_{i, 1} v_{i, 1}+z_{i, 1} \widehat{f}_{i, 1}\left(Z_{i, 1}\right)-z_{i, 1} \sum_{j=1}^{M} a_{i j} x_{j, 2}-\frac{b_{i} \widetilde{\theta}_{i, 1} \dot{\hat{\theta}}_{i, 1}}{\gamma_{i}}
$$

The virtual controller $v_{i, 1}$ is defined as

$$
v_{i, 1}=\frac{1}{d_{i}+h_{i}}\left(-\frac{b_{i} \widehat{\theta}_{i, 1}}{2 \eta_{i, 1}^{2}} S_{i, 1}^{T}\left(Z_{i, 1}\right) S_{i, 1}\left(Z_{i, 1}\right) z_{i, 1}+\sum_{j=1}^{M} a_{i j} x_{j, 2}-b_{i} \varsigma_{i, 13} z_{i, 1}-\varsigma_{i, 11} S_{z_{i, 1}, 1}-\varsigma_{i, 12} S_{z_{i, 1}, 2}\right),
$$

where $\varsigma_{i, 11}, \varsigma_{i, 12}, \varsigma_{i, 13}$, and $\eta_{i, 1}$ are positive design parameters.

In (27), $S_{z_{i, 1}, 1}$ and $S_{z_{i, 1}, 2}$ are defined as

$$
\begin{array}{r}
S_{z_{i, k}, 1}= \begin{cases}\frac{\left(z_{i, k}^{2}\right)^{(1+\alpha) / 2}}{z_{i, k}}, & \left\|z_{i, k}\right\| \geq \varepsilon_{k 0}, \\
\sum_{j=1}^{n_{i}} a_{j}\left(z_{i, k}^{2}\right)^{j}\left(\varepsilon_{k 0}^{2}\right)^{-(j+((1+\alpha) / 2))}, & \left\|z_{i, k}\right\|<\varepsilon_{k 0},\end{cases} \\
S_{z_{i, k}, 2}= \begin{cases}\frac{\left(z_{i, k}^{2}\right)^{(1+\beta) / 2}}{z_{i, k}}, & \left\|z_{i, k}\right\| \geq \varepsilon_{k 0}, \\
\sum_{j=1}^{n_{i}} a_{j}\left(z_{i, k}^{2}\right)^{j}\left(\varepsilon_{k 0}^{2}\right)^{-(j+((1+\beta) / 2))}, & \left\|z_{i, k}\right\|<\varepsilon_{k 0},\end{cases}
\end{array}
$$

where $k=1, \ldots, n_{i}$, and $\varepsilon_{k 0}$ is a positive design parameter, and coefficients $a_{j}, j=1, \ldots, n_{i}$ are calculated using the following equation:

$$
\left[\begin{array}{ccccc}
1 & 1 & \cdots & 1 & 1 \\
1 & 2 & \cdots & n_{i}-1 & n_{i} \\
0 & 2 \times 1 & \cdots & \left(n_{i}-1\right)\left(n_{i}-2\right) & n_{i}\left(n_{i}-1\right) \\
\vdots & \vdots & \ddots & \vdots & \vdots \\
0 & 0 & \cdots & \prod_{j=0}^{n_{i}-2}\left(n_{i}-1-j\right) & \prod_{j=0}^{n_{i}-2}\left(n_{i}-j\right)
\end{array}\right] \times\left[\begin{array}{c}
a_{1} \\
a_{2} \\
a_{3} \\
\vdots \\
a_{n_{i}}
\end{array}\right]=\left[\begin{array}{c}
b_{1} \\
b_{2} \\
b_{3} \\
\vdots \\
b_{n_{i}}
\end{array}\right],
$$

where $b_{1}=1, b_{2}=3 / 4, b_{3}=(3 / 4)((3 / 4)-1), \ldots$, and $b_{n}=$ $\prod_{j=0}^{n_{i}-2}((3 / 4)-j)$

Remark 8. One of the main contributions of this paper is to design suitable virtual controllers $v_{i, k}$ so that nonlinear strictfeedback systems meet the requirement of fixed-time control. In [36-39], virtual controllers designed exhibit similar power functions $z^{2 \alpha-1}$, where $0<\alpha<1$. If $\alpha$ is not appropriately selected, it will make $z$ unsolvable at the origin and in the negative domain. As $0<\alpha<1$, therefore, power exponents $2 \alpha-1 \in(-1,1)$, which leads to the possibility of 
negative power exponents. For example, if $2 \alpha-1=-(1 / 3)$, $z^{-(1 / 3)}$ is unsolvable at $z=0$, that is, $(0)^{-(1 / 3)}$ does not exist. Suppose $2 \alpha-1=\left(q_{1} / q_{2}\right) \in(-1,1)$, where $q_{2}$ is an even number, then $z^{\left(q_{1} / q_{2}\right)}$ is unsolvable at the negative domain. For example, if $\left(q_{1} / q_{2}\right)=1 / 2, z^{1 / 2}$ is unsolvable at the negative domain, that is, $(-1)^{1 / 2}$ does not exist. The controller designed in this paper overcomes the abovementioned defect and promotes the application of fixed-time control in more common nonlinear systems.

Substituting (27) into (26) yields

$$
\dot{V}_{i, 1} \leq\left(d_{i}+h_{i}\right) z_{i, 1} z_{i, 2}+z_{i, 1} \widehat{f}_{i, 1}\left(Z_{i, 1}\right) \frac{b_{i} \widehat{\theta}_{i, 1}}{2 \eta_{i, 1}^{2}} S_{i, 1}^{T}\left(Z_{i, 1}\right) S_{i, 1}\left(Z_{i, 1}\right) z_{i, 1}^{2}-b_{i} \varsigma_{i, 13} z_{i, 1}^{2}-\frac{b_{i} \widetilde{\theta}_{i, 1} \dot{\hat{\theta}}_{i, 1}}{\gamma_{i}}-\varsigma_{i, 11} z_{i, 1} S_{z_{i, 1}, 1}-\varsigma_{i, 12} z_{i, 1} S_{z_{i, 1}, 2}
$$

According to (19) and inequalities (20)-(21), we have

$$
\dot{V}_{i, 1} \leq \frac{b_{i} \widetilde{\theta}_{i, 1}}{\gamma_{i}}\left(\frac{\gamma_{i}}{2 \eta_{i, 1}^{2}} S_{i, 1}^{T}\left(Z_{i, 1}\right) S_{i, 1}\left(Z_{i, 1}\right) z_{i, 1}^{2}-\dot{\hat{\theta}}_{i, 1}\right)+\left(d_{i}+h_{i}\right) z_{i, 1} z_{i, 2}+\sigma_{i, 1}-\varsigma_{i, 11} z_{i, 1} S_{z_{i, 1}, 1}-\varsigma_{i, 12} z_{i, 1} S_{z_{i, 1}, 2}
$$

where $\sigma_{i, 1}=(1 / 2) \eta_{i, 1}^{2}+\left(\left(\varepsilon_{i, 1}^{2}\right) /\left(4 b_{i} \varsigma_{i, 13}\right)\right)$.

The adaptive law $\hat{\theta}_{i, 1}$ is then defined as

$$
\dot{\widehat{\theta}}_{i, 1}=\frac{\gamma_{i}}{2 \eta_{i, 1}^{2}} S_{i, 1}^{T}\left(Z_{i, 1}\right) S_{i, 1}\left(Z_{i, 1}\right) z_{i, 1}^{2}-\lambda_{i} \widehat{\theta}_{i, 1}
$$

where $\lambda_{i}$ with $i=1, \ldots, M$ are positive design parameters.

Combining with Assumption 1 and substituting (33) into (32), we have

$$
\dot{V}_{i, 1} \leq-\varsigma_{i, 11} z_{i, 1} S_{z_{i, 1}, 1}-\varsigma_{i, 12} z_{i, 1} S_{z_{i, 1}, 2}-\varsigma_{i, 11} 2^{(1+\alpha) / 2}\left(\frac{b_{i} \widetilde{\theta}_{i, 1}^{2}}{2 \gamma_{i}}\right)^{(1+\alpha) / 2}-\varsigma_{i, 12} 2^{(1+\beta) / 2}\left(\frac{b_{i} \widetilde{\theta}_{i, 1}^{2}}{2 \gamma_{i}}\right)^{(1+\beta) / 2}+\left(d_{i}+h_{i}\right) z_{i, 1} z_{i, 2}+C_{i, 1}
$$

where $\quad C_{i, 1}=\sigma_{i, 1}+\beta_{i, 1}+\left(\lambda_{i} b_{i} / 2 \gamma_{i}\right) \theta_{i, 1}^{2}, \quad \beta_{i, 1}=\varsigma_{i, 11} 2^{(1+\alpha) / 2}$ $\left(b_{i} Q_{i, 1}^{2} / 2 \gamma_{i}\right)^{(1+\alpha) / 2}+\varsigma_{i, 12} 2^{(1+\beta) / 2}\left(b_{i} Q_{i, 1}^{2} / 2 \gamma_{i}\right)^{(1+\beta) / 2}$, and $\left(\lambda_{i} b_{i} \tilde{\theta}_{i, 1} \widehat{\theta}_{i, 1} / \gamma_{i}\right) \leq\left(\lambda_{i} b_{i} / \gamma_{i}\right)\left(\left(\theta_{i, 1}^{2} / 2\right)-\left(\widetilde{\theta}_{i, 1}^{2} / 2\right)\right) \leq\left(\lambda_{i} b_{i} / 2 \gamma_{i}\right) \theta_{i, 1}^{2}$.
According to (22), (23), (28), and (29), if $\left\|z_{i, 1}\right\| \geq \varepsilon_{10}$, then (34) is rewritten as

$$
\dot{V}_{i, 1} \leq-\varsigma_{i, 11} 2^{1+\alpha / 2}\left(\frac{1}{2} z_{i, 1}^{2}+\frac{b_{i} \widetilde{\theta}_{i, 1}^{2}}{2 \gamma_{i}}\right)^{1+\alpha / 2}-2 \varsigma_{i, 12}\left(\frac{1}{2} z_{i, 1}^{2}+\frac{b_{i} \widetilde{\theta}_{i, 1}^{2}}{2 \gamma_{i}}\right)^{1+\beta / 2}+\left(d_{i}+h_{i}\right) z_{i, 1} z_{i, 2}+C_{i, 1} .
$$

According to (22), (23), (28), and (29), if $\left\|z_{i, 1}\right\|<\varepsilon_{10}$, then (34) is rewritten as

$$
\begin{aligned}
\dot{V}_{i, 1} \leq & \varsigma_{i, 11}\left(z_{i, 1}^{2}\right)^{(1+\alpha) / 2}-\varsigma_{i, 11} z_{i, 1} \sum_{j=1}^{n_{i}} a_{j}\left(z_{i, 1}^{2}\right)^{j}\left(\varepsilon_{10}^{2}\right)^{-j+((1+\alpha) / 2)}+\varsigma_{i, 12}\left(z_{i, 1}^{2}\right)^{(1+\beta) / 2}-\varsigma_{i, 12} z_{i, 1} \sum_{j=1}^{n_{i}} a_{j}\left(z_{i, 1}^{2}\right)^{j}\left(\varepsilon_{10}^{2}\right)^{-j+((1+\beta) / 2)}-\varsigma_{i, 11} 2^{(1+\alpha) / 2}\left(\frac{1}{2} z_{i, 1}^{2}+\frac{b_{i} \widetilde{\theta}_{i, 1}^{2}}{2 \gamma_{i}}\right)^{(1+\alpha) / 2} \\
& -2 \varsigma_{i, 12}\left(\frac{1}{2} z_{i, 1}^{2}+\frac{b_{i} \widetilde{\theta}_{i, 1}^{2}}{2 \gamma_{i}}\right)^{(1+\beta) / 2}+\left(d_{i}+h_{i}\right) z_{i, 1} z_{i, 2}+C_{i, 1} .
\end{aligned}
$$


Remark 9 (see [46]). Based on (28) and (29), when $\left\|z_{i, 1}\right\|<\varepsilon_{10}$, there is an additional term in (36): $\varsigma_{i, 11}\left(z_{i, 1}^{2}\right)^{(1+\alpha) / 2}$ $-\varsigma_{i, 11} z_{i, 1} \sum_{j=1}^{n_{i}} a_{j}\left(z_{i, 1}^{2}\right)^{j}\left(\varepsilon_{10}^{2}\right)^{-j+((1+\alpha) / 2)}+\varsigma_{i, 12}\left(z_{i, 1}^{2}\right)^{(1+\beta) / 2}-$ $\varsigma_{i, 12} z_{i, 1} \sum_{j=1}^{n_{i}} a_{j}\left(z_{i, 1}^{2}\right)^{j}\left(\varepsilon_{10}^{2}\right)^{-j+((1+\beta) / 2)}$. Note that if $\left\|z_{i, 1}\right\|<\varepsilon_{10}$, this additional term is obviously limited by some smaller constant $\varepsilon_{11}$, so the structure of (35) is retained, while the constant term $C_{i, 1}$ only slightly increases. Owing to page limitations and to avoid repetitive discussions, we will omit this part in the rest of the analysis.

Step 2. according to (15) and (18), we have

$$
\dot{z}_{i, 2}=x_{i, 3}+f_{i, 2}\left(\bar{x}_{i, 2}\right)-\dot{v}_{i, 1} \text {, }
$$

where $\dot{v}_{i, 1}=\left(\partial v_{i, 1} / \partial x_{i, 1}\right) \dot{x}_{i, 1}+\sum_{j=1}^{M} a_{i j}\left(\left(\partial v_{i, 1} / \partial x_{j, 1}\right) \dot{x}_{j, 1}+\left(\partial v_{i, 1} /\right.\right.$ $\left.\left.\partial x_{j, 2}\right) \dot{x}_{j, 2}\right)+h_{i}\left(\left(\partial v_{i, 1} / \partial x_{0,1}\right) \dot{x}_{0,1}+\left(\partial v_{i, 1} / \partial x_{0,2}\right) \dot{x}_{0,2}\right)+\left(\partial v_{i, 1} /\right.$ $\left.\partial \widehat{\theta}_{i, 1}\right) \widehat{\theta}_{i, 1}$.

Construct a Lyapunov function as

$$
V_{i, 2}=V_{i, 1}+\frac{1}{2} z_{i, 2}^{2}+\frac{b_{i} \widetilde{\theta}_{i, 2}^{2}}{2 \gamma_{i}}
$$

The derivative of $V_{i, 2}$ is written as

$$
\begin{gathered}
\dot{V}_{i, 2} \leq-\varsigma_{i, 11} 2^{(1+\alpha) / 2}\left(\frac{1}{2} z_{i, 1}^{2}+\frac{b_{i} \widetilde{\theta}_{i, 1}^{2}}{2 \gamma_{i}}\right)^{(1+\alpha) / 2}-2 \varsigma_{i, 12}\left(\frac{1}{2} z_{i, 1}^{2}+\frac{b_{i} \widetilde{\theta}_{i, 1}^{2}}{2 \gamma_{i}}\right)^{(1+\beta) / 2}+z_{i, 2} \widehat{f}_{i, 2}\left(Z_{i, 2}\right)+z_{i, 2} z_{i, 3}+ \\
z_{i, 2} \nu_{i, 2}-z_{i, 2} \frac{\partial v_{i, 1}}{\partial \widehat{\theta}_{i, 1}} \dot{\widehat{\theta}}_{i, 1}-\frac{b_{i} \widetilde{\theta}_{i, 2} \dot{\widehat{\theta}}_{i, 2}}{\gamma_{i}}+z_{i, 2} M_{i, 1}\left(Z_{i, 2}\right)+C_{i, 1},
\end{gathered}
$$

where

$$
\widehat{f}_{i, 2}\left(Z_{i, 2}\right)=f_{i, 2}+z_{i, 1}\left(d_{i}+h_{i}\right)-\frac{\partial v_{i, 1}}{\partial x_{i, 1}} \dot{x}_{i, 1}-\sum_{j=1}^{M} a_{i j}\left(\frac{\partial v_{i, 1}}{\partial x_{j, 1}} \dot{x}_{j, 1}+\frac{\partial v_{i, 1}}{\partial x_{j, 2}} \dot{x}_{j, 2}\right)-h_{i}\left(\frac{\partial v_{i, 1}}{\partial x_{0,1}} \dot{x}_{0,1}+\frac{\partial v_{i, 1}}{\partial x_{0,2}} \dot{x}_{0,2}\right)-M_{i, 1}\left(Z_{i, 2}\right)
$$

Remark 10. $M_{i, 1}\left(Z_{i, 2}\right)$ is a smooth function that is used to overcome the design difficulty of $\left(\left(\partial v_{i, 1}\right) /\left(\partial \widehat{\theta}_{i, 1}\right)\right) \widehat{\theta}_{i, 1}$.

The virtual controller $v_{i, 2}$ is defined as

$v_{i, 2}=-\varsigma_{i, 21} S_{z_{i, 2}, 1}-\varsigma_{i, 22} S_{z_{i, 2}, 2}-\frac{b_{i} \widehat{\theta}_{i, 2}}{2 \eta_{i, 2}^{2}} S_{i, 2}^{T}\left(Z_{i, 2}\right) S_{i, 2}\left(Z_{i, 2}\right) z_{i, 2}-b_{i} \varsigma_{i, 23} z_{i, 2}$

where $\varsigma_{i, 21}, \varsigma_{i, 22}, \varsigma_{i, 23}$, and $\eta_{i, 2}$ are positive design parameters.

$$
\begin{aligned}
\dot{V}_{i, 2} \leq & -\varsigma_{i, 21} z_{i, 2} S_{z_{i, 2}, 1}-\varsigma_{i, 22} z_{i, 2} S_{z_{i, 2}, 2}-\varsigma_{i, 21} 2^{(1+\alpha) / 2}\left(\frac{b_{i} \widetilde{\theta}_{i, 2}^{2}}{2 \gamma_{i}}\right)^{(1+\alpha) / 2}-\varsigma_{i, 22} 2^{(1+\beta) / 2}\left(\frac{b_{i} \widetilde{\theta}_{i, 2}^{2}}{2 \gamma_{i}}\right)^{(1+\beta) / 2}-\varsigma_{i, 11} 2^{(1+\alpha) / 2}\left(\frac{1}{2} z_{i, 1}^{2}+\frac{b_{i} \widetilde{\theta}_{i, 1}^{2}}{2 \gamma_{i}}\right)^{(1+\alpha) / 2} \\
& -2 \varsigma_{i, 12}\left(\frac{1}{2} z_{i, 1}^{2}+\frac{b_{i} \tilde{\theta}_{i, 1}^{2}}{2 \gamma_{i}}\right)^{(1+\beta) / 2} \\
& \sum_{j=1}^{2} C_{i, j}+z_{i, 2} z_{i, 3}+z_{i, 2}\left(M_{i, 1}\left(Z_{i, 2}\right)-\frac{\partial \nu_{i, 1}}{\partial \widehat{\theta}_{i, 1}} \hat{\theta}_{i, 1}\right)
\end{aligned}
$$

where $C_{i, 2}=\sigma_{i, 2}+\beta_{i, 2}+\left(\lambda_{i} b_{i} / 2 \gamma_{i}\right) \theta_{i, 2}^{2}, \quad \beta_{i,} \quad 2=\varsigma_{i, 21} 2^{(1+\alpha) / 2}$ $\left(b_{i} Q_{i, 2}^{2} / 2 \gamma_{i}\right)^{(1+\alpha) / 2}+c_{i, 22} 2^{(1+\beta) / 2}\left(b_{i} Q_{i, 2}^{2} / 2 \gamma_{i}\right)^{(1+\beta) / 2}, \quad \sigma_{i, 2}=$
Choose the adaptive law $\widehat{\theta}_{i, 2}$ as

$$
\dot{\widehat{\theta}}_{i, 2}=\frac{\gamma_{i}}{2 \eta_{i, 2}^{2}} S_{i, 2}^{T}\left(Z_{i, 2}\right) S_{i, 2}\left(Z_{i, 2}\right) z_{i, 2}^{2}-\lambda_{i} \widehat{\theta}_{i, 2}
$$

Combining (19)-(21) and Assumption 1 and substituting (41) and (42) into (38) and adopting the same design method as in Step 1 yields 
According to (22), (23), and Remark 9, we consider only $\left\|z_{i, 2}\right\| \geq \varepsilon_{20}$. Then, (43) can be written as

$$
\left.\dot{V}_{i, 2} \leq-2^{(1+\alpha) / 2} \sum_{j=1}^{2} \varsigma_{i, j 1}\left(\frac{1}{2} z_{i, j}^{2}+\frac{b_{i} \widetilde{\theta}_{i, j}^{2}}{2 \gamma_{i}}\right)^{(1+\alpha) / 2}-2 \sum_{j=1}^{2} \varsigma_{i, j 2}\left(\frac{1}{2} z_{i, j}^{2}+\frac{b_{i} \widetilde{\theta}_{i, j}^{2}}{2 \gamma_{i}}\right)^{(1+\beta) / 2}+\sum_{j=1}^{2} C_{j}+z_{i, 2} z_{i, 3}+z_{i, 2}\left(M_{i, 1}\left(Z_{i, 2}\right)-\frac{\partial v_{i, 1}}{\partial \widehat{\hat{\theta}}_{i, 1}}\right)_{i, 1}\right)
$$

It can be seen from (44) that defining the design smooth function $M_{i, 1}\left(Z_{i, 2}\right)$ to overcome the design difficulty of $\left(\partial v_{i, 1} / \partial \widehat{\theta}_{i, 1}\right) \widehat{\theta}_{i, 1}$ is one of the difficulties of designing the that

From Lemma 1, Lemma 5, Lemma 6, and (33), it follows controllers in this paper.

$$
\begin{aligned}
& -z_{i, 2} \frac{\partial v_{i, 1}}{\partial \widehat{\theta}_{i, 1}} \widehat{\theta}_{i, 1} \leq-z_{i, 2} \frac{\partial v_{i, 1}}{\partial \widehat{\theta}_{i, 1}}\left(\frac{\gamma_{i}}{2 \eta_{i, 1}^{2}} S_{i, 1}^{T}\left(Z_{i, 1}\right) S_{i, 1}\left(Z_{i, 1}\right) z_{i, 1}^{2}-\lambda_{i} \widehat{\theta}_{i, 1}\right) \leq \frac{z_{i, 2}^{2}\left(\partial v_{i, 1} / \partial \widehat{\theta}_{i, 1}\right)^{2}\left(\left(\gamma_{i} / 2 \eta_{i, 1}^{2}\right) S_{i, 1}^{T}\left(Z_{i, 1}\right) S_{i, 1}\left(Z_{i, 1}\right) z_{i, 1}^{2}\right)^{2}}{\sqrt{z_{i, 2}^{2}\left(\partial v_{i, 1} / \partial \widehat{\theta}_{i, 1}\right)^{2}\left(\left(\gamma_{i} / 2 \eta_{i, 1}^{2}\right) S_{i, 1}^{T}\left(Z_{i, 1}\right) S_{i, 1}\left(Z_{i, 1}\right) z_{i, 1}^{2}\right)^{2}+\kappa^{2}}} \\
& +z_{i, 2} \frac{\partial \nu_{i, 1}}{\partial \widehat{\theta}_{i, 1}} \lambda_{i} \widehat{\theta}_{i, 1} \leq \frac{z_{i, 2}^{2}\left(\partial \nu_{i, 1} / \partial \widehat{\theta}_{i, 1}\right)^{2}\left(\left(\gamma_{i} / 2 \eta_{i, 1}^{2}\right) S^{2} z_{i, 1}^{2}\right)^{2}}{\sqrt{z_{i, 2}^{2}\left(\partial v_{i, 1} / \partial \widehat{\theta}_{i, 1}\right)^{2}\left(\left(\gamma_{i} / 2 \eta_{i, 1}^{2}\right) S^{2} z_{i, 1}^{2}\right)^{2}+\kappa^{2}}}+z_{i, 2} \frac{\partial v_{i, 1}}{\partial \widehat{\theta}_{i, 1}} \lambda_{i} \widehat{\theta}_{i, 1} .
\end{aligned}
$$

Therefore, $M_{i, 1}\left(Z_{i, 2}\right)$ can be defined as

$M_{i, 1}\left(Z_{i, 2}\right)=-\frac{z_{i, 2}\left(\partial v_{i, 1} / \partial \widehat{\theta}_{i, 1}\right)^{2}\left(\left(\gamma_{i} / 2 \eta_{i, 1}^{2}\right) S^{2} z_{i, 1}^{2}\right)^{2}}{\sqrt{z_{i, 2}^{2}\left(\partial v_{i, 1} / \partial \widehat{\theta}_{i, 1}\right)^{2}\left(\left(\gamma_{i} / 2 \eta_{i, 1}^{2}\right) S^{2} z_{i, 1}^{2}\right)^{2}+\kappa^{2}}}-\frac{\partial v_{i, 1}}{\partial \widehat{\theta}_{i, 1}} \lambda_{i} \widehat{\theta}_{i, 1}$

with the result that

$$
z_{i, 2}\left(M_{i, 1}\left(Z_{i, 2}\right)-\frac{\partial v_{i, 1}}{\partial \widehat{\theta}_{i, 1}}\right) \leq 0
$$

Substituting (47) into (44) yields

$$
\dot{V}_{i, 1} \leq-2^{(1+\alpha) / 2} \sum_{j=1}^{2} \varsigma_{i, j 1}\left(\frac{1}{2} z_{i, j}^{2}+\frac{b_{i} \tilde{\theta}_{i, j}^{2}}{2 \gamma_{i}}\right)^{(1+\alpha) / 2}-2 \sum_{j=1}^{2} \varsigma_{i, j 2}\left(\frac{1}{2} z_{i, j}^{2}+\frac{b_{i} \tilde{\theta}_{i, j}^{2}}{2 \gamma_{i}}\right)^{(1+\beta) / 2}+\sum_{j=1}^{2} C_{i, j}+z_{i, 2} z_{i, 3} .
$$

Step $k\left(3 \leq k \leq n_{i}-1\right)$ : according to (15) and (18), we have

$$
\dot{z}_{i, k}=x_{i, k+1}+f_{i, k}\left(\bar{x}_{i, k}\right)-\dot{v}_{i, k-1} \text {, }
$$

where $\dot{v}_{i, k-1}=\sum_{j=1}^{k-1}\left(\partial v_{i, k-1} / \partial x_{i, j}\right) \dot{x}_{i, j}+\sum_{j=1}^{M} a_{i j} \sum_{q=1}^{k}\left(\left(\partial v_{i, k-1} /\right.\right.$ $\left.\left.\partial x_{i, q}\right) \dot{x}_{j, q}\right)+h_{i} \sum_{j=1}^{k}\left(\partial v_{i, k-1} / \partial x_{0, j}\right) \dot{x}_{0, j}+\sum_{j=1}^{k-1}\left(\partial v_{i, k-1} / \partial \widehat{\theta}_{i, j}\right) \widehat{\theta}_{i, j}$.
Construct a Lyapunov function as

$$
V_{i, k}=V_{i, k-1}+\frac{1}{2} z_{i, k}^{2}+\frac{b_{i} \tilde{\theta}_{i, k}^{2}}{2 \gamma_{i}} .
$$

The derivative of $V_{i, k}$ is written as

$$
\begin{aligned}
\dot{V}_{i, k} \leq & -2^{(1+\alpha) / 2} \sum_{j=1}^{k-1} \varsigma_{i, j 1}\left(\frac{1}{2} z_{i, j}^{2}+\frac{b_{i} \widetilde{\theta}_{i, j}^{2}}{2 \gamma_{i}}\right)^{(1+\alpha) / 2}-2 \sum_{j=1}^{k-1} \varsigma_{i, j 2}\left(\frac{1}{2} z_{i, j}^{2}+\frac{b_{i} \widetilde{\theta}_{i, j}^{2}}{2 \gamma_{i}}\right)^{(1+\alpha) / 2}+z_{i, k} \widehat{f}_{i, k}\left(Z_{i, k}\right) \\
& +z_{i, k} z_{i, k+1}+z_{i, k} \nu_{i, k}-z_{i, k} \sum_{j=1}^{k-1} \frac{\partial \nu_{i, k-1}}{\partial \widehat{\theta}_{i, j}} \hat{\theta}_{i, j}-\frac{b_{i} \widetilde{\theta}_{i, k} \hat{\widehat{\theta}}_{i, k}}{\gamma_{i}}+z_{i, k} M_{i, k-1}\left(Z_{i, k}\right)+\sum_{j=1}^{k-1} C_{i, j},
\end{aligned}
$$


where

$$
\begin{aligned}
\hat{f}_{i, k}\left(Z_{i, k}\right)= & f_{i, k}\left(\bar{x}_{i, k}\right)-\sum_{j=1}^{k-1} \frac{\partial v_{i, k-1}}{\partial x_{i, j}} \dot{x}_{i, j}-\sum_{j=1}^{M} a_{i j} \sum_{q=1}^{k}\left(\frac{\partial v_{i, k-1}}{\partial x_{j, q}} \dot{x}_{j, q}\right)-h_{i} \sum_{j=1}^{k} \frac{\partial v_{i, k-1}}{\partial x_{0, j}} \dot{x}_{0, j} \\
& +z_{i, k-1}-M_{i, k-1}\left(Z_{i, k}\right) .
\end{aligned}
$$

Defining virtual controller $v_{i, k}$ as

$v_{i, k}=-\varsigma_{i, k 1} S_{z_{i, k}, 1}-\varsigma_{i, k 2} S_{z_{i, k}, 2}-\frac{b_{i} \hat{\theta}_{i, k}}{2 \eta_{i, k}^{2}} S_{i, k}^{T}\left(Z_{i, k}\right) S_{i, k}\left(Z_{i, k}\right) z_{i, k}-b_{i} \varsigma_{i, k 3} z_{i, k}$,

where $\varsigma_{i, k 1}, \varsigma_{i, k 2}, \varsigma_{i, k 3}$, and $\eta_{i, k}$ are positive design parameters.
Choose the adaptive law $\widehat{\theta}_{i, k}$ as

$$
\dot{\hat{\theta}}_{i, k}=\frac{\gamma_{i}}{2 \eta_{i, \mathrm{k}}^{2}} S_{i, \mathrm{k}}^{T}\left(Z_{i, \mathrm{k}}\right) S_{i, \mathrm{k}}\left(Z_{i, \mathrm{k}}\right) z_{i, \mathrm{k}}^{2}-\lambda_{i} \widehat{\theta}_{i, k} .
$$

Combining (19)-(21) and Assumption 1 and substituting (53) and (54) into (51) yields

$$
\begin{aligned}
\dot{V}_{i, k} \leq & -2^{(1+\alpha) / 2} \sum_{j=1}^{k-1} \varsigma_{i, j 1}\left(\frac{1}{2} z_{i, j}^{2}+\frac{b_{i} \tilde{\theta}_{i, j}^{2}}{2 \gamma_{i}}\right)^{(1+\alpha) / 2}-2 \sum_{j=1}^{k-1} \varsigma_{i, j 2}\left(\frac{1}{2} z_{i, j}^{2}+\frac{b_{i} \widetilde{\theta}_{i, j}^{2}}{2 \gamma_{i}}\right)^{(1+\beta) / 2}-c_{i, k 1} z_{i, k} S_{z_{i, k}, 1}-c_{i, k 2} z_{i, k} S_{z_{i, k}, 2}-c_{i, k 1} 2^{(1+\alpha) / 2}\left(\frac{b_{i} \tilde{\theta}_{i, \mathrm{k}}^{2}}{2 \gamma_{i}}\right)^{(1+\alpha) / 2} \\
& +z_{i, k} z_{i, k+1}+\sum_{j=1}^{k} C_{i, j} \\
& -\varsigma_{i, k 2} 2^{(1+\beta) / 2}\left(\frac{b_{i} \widetilde{\theta}_{i, \mathrm{k}}^{2}}{2 \gamma_{i}}\right)^{(1+\beta) / 2}+z_{i, k}\left(M_{i, k-1}\left(Z_{i, k}\right)-\sum_{j=1}^{k-1} \frac{\partial v_{i, k-1}}{\partial \widetilde{\theta}_{i, j}} \hat{\theta}_{i, j}\right),
\end{aligned}
$$

where $C_{i, k}=\sigma_{i, k}+\beta_{i, k}+\left(\lambda_{i} b_{i} / 2 \gamma_{i}\right) \theta_{i, k}^{2}, \quad \beta_{i, k}=\varsigma_{i}, \quad k 12^{(1+\alpha) / 2}$ $\left(b_{i} Q_{i, k}^{2} / 2 \gamma_{i}\right)^{(1+\alpha) / 2}+\varsigma_{i, k 2} 2^{(1+\beta) / 2}\left(b_{i} Q_{i, k}^{2} / 2 \gamma_{i}\right)^{(1+\beta) / 2}, \sigma_{i, k}=(1 / 2)$ $\eta_{i, k_{2}}^{2}+\left(\varepsilon_{i, k}^{2} / 4 b_{i} \varsigma_{i, k 3}\right)$, and $\left(\lambda_{i} b_{i} \widetilde{\theta}_{i, k} \widehat{\theta}_{i, k} / \gamma_{i}\right) \leq\left(\lambda_{i} b_{i} / \gamma_{i}\right)\left(\left(\theta_{i, k}^{2} / 2\right)-\right.$ $\left.\left(\hat{\theta}_{i, k}^{2} / 2\right)\right) \leq\left(\lambda_{i} b_{i} / 2 \gamma_{i}\right) \theta_{i, k}^{2}$.
According to (22), (23), and Remark 9, we consider only $\left\|z_{i, k}\right\| \geq \varepsilon_{k 0}$. Then, (55) can be written as

$$
\begin{aligned}
\dot{V}_{i, k} \leq & -2^{(1+\alpha) / 2} \sum_{j=1}^{k-1} \varsigma_{i, j 1}\left(\frac{1}{2} z_{i, j}^{2}+\frac{b_{i} \widetilde{\theta}_{i, j}^{2}}{2 \gamma_{i}}\right)^{(1+\alpha) / 2}-2 \sum_{j=1}^{k-1} \varsigma_{i, j 2}\left(\frac{1}{2} z_{i, j}^{2}+\frac{b_{i} \widetilde{\theta}_{i, j}^{2}}{2 \gamma_{i}}\right)^{(1+\beta) / 2} \\
& +\sum_{j=1}^{k} C_{j}+z_{i, k} z_{i, k+1}+z_{i, k}\left(M_{i, k-1}\left(Z_{i, k}\right)-\sum_{j=1}^{k-1} \frac{\partial v_{i, k-1}}{\partial \widehat{\theta}_{i, j}} \dot{\hat{\theta}}_{i, j}\right) .
\end{aligned}
$$


From Lemma 1, Lemma 5, Lemma 6, and (54), it follows that

$$
\begin{aligned}
& -z_{i, k} \sum_{j=1}^{k-1} \frac{\partial \nu_{i, k-1}}{\partial \widehat{\theta}_{i, j}} \dot{\theta}_{i, j} \leq-z_{i, k} \sum_{j=1}^{k-1} \frac{\partial \nu_{i, k-1}}{\partial \widehat{\theta}_{i, j}}\left(\frac{\gamma_{i}}{2 \eta_{i, j}^{2}} S_{i, j}^{T}\left(Z_{i, j}\right) S_{i, j}\left(Z_{i, j}\right) z_{i, j}^{2}-\lambda_{i} \widehat{\theta}_{i, j}\right) \\
& \leq \sum_{j=1}^{k-1} \frac{z_{i, k}^{2}\left(\partial v_{i, k-1} / \partial \widehat{\theta}_{i, j}\right)^{2}\left(\left(\gamma_{i} / 2 \eta_{i, j}^{2}\right) S_{i, j}^{T}\left(Z_{i, j}\right) S_{i, j}\left(Z_{i, j}\right) z_{i, j}^{2}\right)^{2}}{\sqrt{z_{i, k}^{2}\left(\partial v_{i, k-1} / \partial \widehat{\theta}_{i, j}\right)^{2}\left(\left(\gamma_{i} / 2 \eta_{i, j}^{2}\right) S_{i, j}^{T}\left(Z_{i, j}\right) S_{i, j}\left(Z_{i, j}\right) z_{i, j}^{2}\right)^{2}+\kappa^{2}}}+\lambda_{i} z_{i, k} \sum_{j=1}^{k-1} \frac{\partial v_{i, k-1}}{\partial \widehat{\theta}_{i, j}} \widehat{\theta}_{i, j} \\
& \leq \sum_{j=1}^{k-1} \frac{z_{i, k}^{2}\left(\partial v_{i, k-1} / \partial \widehat{\theta}_{i, j}\right)^{2}\left(\left(\gamma_{i} / 2 \eta_{i, j}^{2}\right) S^{2} z_{i, j}^{2}\right)^{2}}{\sqrt{z_{i, k}^{2}\left(\partial v_{i, k-1} / \partial \widehat{\theta}_{i, j}\right)^{2}\left(\left(\gamma_{i} / 2 \eta_{i, j}^{2}\right) S^{2} z_{i, j}^{2}\right)^{2}+\kappa^{2}}}+\lambda_{i} z_{i, k} \sum_{j=1}^{k-1} \frac{\partial v_{i, k-1}}{\partial \widehat{\theta}_{i, j}} \widehat{\theta}_{i, j}
\end{aligned}
$$

Therefore, $M_{i, k-1}\left(Z_{i, k}\right)$ can be defined as

$$
M_{i, k-1}\left(Z_{i, k}\right)=-\sum_{j=1}^{k-1} \frac{z_{i, k}^{2}\left(\partial v_{i, k-1} / \partial \widehat{\theta}_{i, j}\right)^{2}\left(\left(\gamma_{i} / 2 \eta_{i, 1}^{2}\right) S^{2} z_{i, 1}^{2}\right)^{2}}{\sqrt{z_{i, k}^{2}\left(\partial v_{i, k-1} / \partial \widehat{\theta}_{i, j}\right)^{2}\left(\left(\gamma_{i} / 2 \eta_{i, j}^{2}\right) S^{2} z_{i, j}^{2}\right)^{2}+\kappa^{2}}}+\lambda_{i} z_{i, k} \sum_{j=1}^{k-1} \frac{\partial v_{i, k-1}}{\partial \widehat{\theta}_{i, j}} \widehat{\theta}_{i, j}
$$

with the result that

Substituting (59) into (56) yields

$$
z_{i, k}\left(M_{i, k-1}\left(Z_{i, k}\right)-\sum_{j=1}^{k-1} \frac{\left.\partial v_{i, k-1} \hat{\theta}_{i, j}\right) \leq 0}{\partial \widehat{\theta}_{i, j}}\right.
$$

$$
\dot{V}_{i, k} \leq-2^{(1+\alpha) / 2} \sum_{j=1}^{k-1} \varsigma_{i, j 1}\left(\frac{1}{2} z_{i, j}^{2}+\frac{b_{i} \widetilde{\theta}_{i, j}^{2}}{2 \gamma_{i}}\right)^{(1+\alpha) / 2}-2 \sum_{j=1}^{k-1} \varsigma_{i, j 2}\left(\frac{1}{2} z_{i, j}^{2}+\frac{b_{i} \widetilde{\theta}_{i, j}^{2}}{2 \gamma_{i}}\right)^{(1+\beta) / 2}+\sum_{j=1}^{k} C_{j}+z_{i, k} z_{i, k+1} .
$$

Step $n_{i}$ : according to (15) and (18), we have

$$
\dot{z}_{i, n_{i}}=u_{i}+f_{i, n_{i}}\left(x_{i}\right)-\dot{v}_{i, n_{i}-1} \text {, }
$$

where $\dot{v}_{i, n_{i}}-1=\sum_{j=1}^{n_{i}-1}\left(\partial v_{i, n_{i}-1} / \partial x_{i}, j\right) \dot{x}_{i, j}+\sum_{j=1}^{M} a_{i j} \sum_{q=1}^{n_{i}}((\partial$ $\left.\left.v_{i, n_{i}-1} / \partial x_{j, q}\right) \dot{x}_{j, q}\right)+h_{i} \sum_{j=1}^{n_{i}}\left(\left(\partial v_{i, n_{i}-1} / \partial x_{0, j}\right) \dot{x}_{0, j}\right)+\sum_{j=1}^{n_{i}-1}\left(\partial v_{i, n_{i}-1} /\right.$ $\left.\partial \widehat{\theta}_{i, j}\right) \widehat{\theta}_{i, j}$.

\section{Construct a Lyapunov function as}

$$
V_{i, n_{i}}=V_{i, n_{i}-1}+\frac{1}{2} z_{i, n_{i}}^{2}+\frac{b_{i} \widetilde{\theta}_{i, n_{i}}^{2}}{2 \gamma_{i}}
$$

The derivative of $V_{i, n_{i}}$ is written as

$$
\begin{aligned}
\dot{V}_{i, n_{i}} \leq & -2^{(1+\alpha) / 2} \sum_{j=1}^{n_{i}-1} \varsigma_{i, j 1}\left(\frac{1}{2} z_{i, j}^{2}+\frac{b_{i} \widetilde{\theta}_{i, j}^{2}}{2 \gamma_{i}}\right)^{(1+\alpha) / 2}-2 \sum_{j=1}^{n_{i}-1} \varsigma_{i, j 2}\left(\frac{1}{2} z_{i, j}^{2}+\frac{b_{i} \widetilde{\theta}_{i, j}^{2}}{2 \gamma_{i}}\right)^{(1+\beta) / 2}+z_{i, n_{i}} \widehat{f}_{i, n_{i}}\left(Z_{i, n_{i}}\right) \\
& +z_{i, n_{i}} u_{i}-z_{i, n_{i}} \sum_{j=1}^{n_{i}-1} \frac{\partial v_{i, n_{i}-1}}{\partial \dot{\hat{\theta}}_{i, j}}-\frac{b_{i} \widetilde{\theta}_{i, n_{i}} \dot{\widehat{\theta}}_{i, n_{i}}}{\gamma_{i}}+z_{i, n_{i}} M_{i, n_{i}-1}\left(Z_{i, n_{i}}\right)+\sum_{j=1}^{n_{i}-1} C_{i, j},
\end{aligned}
$$


where

$$
\begin{aligned}
\widehat{f}_{i, k}\left(Z_{i, n_{i}}\right)= & f_{i, n_{i}}\left(x_{i}\right)-\sum_{j=1}^{n_{i}-1} \frac{\partial v_{i, n_{i}-1}}{\partial x_{i, j}} \dot{x}_{i, j}-\sum_{j=1}^{M} a_{i j} \sum_{q=1}^{n_{i}}\left(\frac{\partial v_{i, n_{i}-1}}{\partial x_{j, q}} \dot{x}_{j, q}\right)-h_{i} \sum_{j=1}^{n_{i}} \frac{\partial v_{i, n_{i}-1}}{\partial x_{0, j}} \dot{x}_{0, j} \\
& +z_{i, n_{i}-1}-M_{i, n_{i}-1}\left(Z_{i, n_{i}}\right) .
\end{aligned}
$$

Defining virtual controller $u_{i}$ as

$$
\begin{aligned}
u_{i}= & -\varsigma_{i, n_{i} 1} S_{z_{i, n_{i}}, 1}-\varsigma_{i, n_{i}} S_{z_{i, n_{i}}, 2}-\frac{b_{i} \widehat{\theta}_{i, n_{i}}}{2 \eta_{i, n_{i}}^{2}} S_{i, n_{i}}^{T}\left(Z_{i, n_{i}}\right) S_{i, n_{i}}\left(\left(Z_{i, n_{i}}\right) z_{i, n_{i}}\right. \\
& -b_{i} \varsigma_{i, n_{i} 3} z_{i, n_{i}},
\end{aligned}
$$

where $\varsigma_{i, n_{i} 1}, \quad \varsigma_{i, n_{i} 2}, \quad \varsigma_{i, n_{i} 3}$, and $\eta_{i, n_{i}}$ are positive design parameters.

Choose the adaptive law $\widehat{\theta}_{i, n_{i}}$ as

$$
\dot{\hat{\theta}}_{i, n_{i}}=\frac{\gamma_{i}}{2 \eta_{i, n_{i}}^{2}} S_{i, n_{i}}^{T}\left(Z_{i, n_{i}}\right) S_{i, n_{i}}\left(Z_{i, n_{i}}\right) z_{i, n_{i}}^{2}-\lambda_{i} \widehat{\theta}_{i, n_{i}} \text {. }
$$

Combining (19)-(21) and Assumption 1 and substituting (65) and (66) into (63) yields

$$
\begin{aligned}
\dot{V}_{i, n_{i}} \leq & -2^{(1+\alpha) / 2} \sum_{j=1}^{n_{i}-1} \varsigma_{i, j 1}\left(\frac{1}{2} z_{i, j}^{2}+\frac{b_{i} \widetilde{\theta}_{i, j}^{2}}{2 \gamma_{i}}\right)^{(1+\alpha) / 2}-2 \sum_{j=1}^{n_{i}-1} \varsigma_{i, j 2}\left(\frac{1}{2} z_{i, j}^{2}+\frac{b_{i} \widetilde{\theta}_{i, j}^{2}}{2 \gamma_{i}}\right)^{(1+\beta) / 2} \\
& -\varsigma_{i, n_{i}} z_{i, n_{i}} S_{z_{i, n_{i}}, 1}-\varsigma_{i, n_{i}} z_{i, n_{i}} S_{z_{i, n_{i}} 2}-\varsigma_{i, n_{i}} 1^{(1+\alpha) / 2}\left(\frac{b_{i} \widetilde{\theta}_{i, n_{i}}^{2}}{2 \gamma_{i}}\right)^{(1+\alpha) / 2}+\sum_{j=1}^{k} C_{i, j} \\
& -\varsigma_{i, n_{i} 2^{2}} 2^{(1+\beta) / 2}\left(\frac{b_{i} \widetilde{\theta}_{i, n_{i}}^{2}}{2 \gamma_{i}}\right)^{(1+\beta) / 2}+z_{i, n_{i}}\left(M_{i, n_{i}-1}\left(Z_{i, n_{i}}\right)-\sum_{j=1}^{n_{i}-1} \frac{\partial v_{i, n_{i}-1}}{\partial \widehat{\theta}_{i, j}}\right)
\end{aligned}
$$

where $C_{i, n_{i}}=\sigma_{i, n_{i}}+\beta_{i, n_{i}}+\left(\lambda_{i} b_{i} / 2 \gamma_{i}\right) \theta_{i, n_{i}}^{2}, \beta_{i, \mathrm{n}_{i}}=\varsigma_{i, \mathrm{n}_{i}} 2^{(1+\alpha) / 2}$ $\left(b_{i} Q_{i, \mathrm{n}_{i}}^{2} / 2 \gamma_{i}\right)^{(1+\alpha) / 2}+c_{i, \mathrm{n}_{i} 2^{2}} 2^{(1+\beta) / 2}\left(b_{i} Q_{i, \mathrm{n}_{i}}^{2} / 2 \gamma_{i}\right)^{(1+\beta) / 2}, \quad \sigma_{i, n_{i}}=$ According to (22), (23), and Remark 9, we consider only $(1 / 2) \eta_{i, n_{i}}^{2}+\left(\varepsilon_{i, n_{i}}^{2} / 4 b_{i} \varsigma_{i, n_{i}}\right)$, and $\left(\lambda_{i} b_{i} \widetilde{\theta}_{i, \mathrm{n}_{i}} \widehat{\theta}_{i, \mathrm{n}_{i}} / \gamma_{i}\right) \leq\left(\lambda_{i} b_{i} / \gamma_{i}\right)$ $\left(\left(\theta_{i, \mathrm{n}_{i}}^{2} / 2\right)-\left(\tilde{\theta}_{i, \mathrm{n}_{i}}^{2} / 2\right)\right) \leq\left(\lambda_{i} b_{i} / 2 \gamma_{i}\right) \theta_{i, \mathrm{n}_{i}}^{2}$.

$$
\begin{aligned}
\dot{V}_{i, n_{i}} \leq & -2^{(1+\alpha) / 2} \sum_{j=1}^{n_{i}-1} \varsigma_{i, j 1}\left(\frac{1}{2} z_{i, j}^{2}+\frac{b_{i} \widetilde{\theta}_{i, j}^{2}}{2 \gamma_{i}}\right)^{(1+\alpha) / 2}-2 \sum_{j=1}^{n_{i}-1} \varsigma_{i, j 2}\left(\frac{1}{2} z_{i, j}^{2}+\frac{b_{i} \widetilde{\theta}_{i, j}^{2}}{2 \gamma_{i}}\right)^{(1+\beta) / 2} \\
& +\sum_{j=1}^{n_{i}} C_{i, j}+z_{i, n_{i}}\left(M_{i, n_{i}-1}\left(Z_{i, n_{i}}\right)-\sum_{j=1}^{n_{i}-1} \frac{\partial v_{i, n_{i}-1}}{\partial \widehat{\theta}_{i, j}} \widehat{\hat{\theta}}_{i, j}\right) .
\end{aligned}
$$


From Lemma 1, Lemma 5, Lemma 6, and (54), it follows that

$$
\begin{aligned}
& -z_{i, n_{i}} \sum_{j=1}^{n_{i}-1} \frac{\partial v_{i, n_{i}-1}}{\partial \widehat{\theta}_{i, j}} \dot{\hat{\theta}}_{i, j} \leq-z_{i, n_{i}} \sum_{j=1}^{n_{i}-1} \frac{\partial v_{i, n_{i}-1}}{\partial \widehat{\theta}_{i, j}}\left(\frac{\gamma_{i}}{2 \eta_{i, j}^{2}} S_{i, j}^{T}\left(Z_{i, j}\right) S_{i, j}\left(Z_{i, j}\right) z_{i, j}^{2}-\lambda_{i} \widehat{\theta}_{i, j}\right) \\
& \leq \sum_{j=1}^{n_{i}-1} \frac{z_{i, n_{i}}^{2}\left(\partial \nu_{i, n_{i}-1} / \partial \widehat{\theta}_{i, j}\right)^{2}\left(\left(\gamma_{i} / 2 \eta_{i, j}^{2}\right) S_{i, j}^{T}\left(Z_{i, j}\right) S_{i, j}\left(Z_{i, j}\right) z_{i, j}^{2}\right)^{2}}{\sqrt{z_{i, n_{i}}^{2}\left(\partial v_{i, n_{i}-1} / \partial \hat{\theta}_{i, j}\right)^{2}\left(\left(\gamma_{i} / 2 \eta_{i, j}^{2}\right) S_{i, j}^{T}\left(Z_{i, j}\right) S_{i, j}\left(Z_{i, j}\right) z_{i, j}^{2}\right)^{2}+\kappa^{2}}}+\lambda_{i} z_{i, n_{i}} \sum_{j=1}^{n_{i}-1} \frac{\partial \nu_{i, n_{i}-1}}{\partial \widehat{\theta}_{i, j}} \widehat{\theta}_{i, j} \\
& \leq \sum_{j=1}^{n_{i}-1} \frac{z_{i, n_{i}}^{2}\left(\partial v_{i, n_{i}-1} / \partial \widehat{\theta}_{i, j}\right)^{2}\left(\left(\gamma_{i} / 2 \eta_{i, j}^{2}\right) S^{2} z_{i, j}^{2}\right)^{2}}{\sqrt{z_{i, k}^{2}\left(\partial v_{i, n_{i}-1} / \partial \widehat{\theta}_{i, j}\right)^{2}\left(\left(\gamma_{i} / 2 \eta_{i, j}^{2}\right) S^{2} z_{i, j}^{2}\right)^{2}+\kappa^{2}}}+\lambda_{i} z_{i, n_{i}} \sum_{j=1}^{n_{i}-1} \frac{\partial v_{i, n_{i}-1}}{\partial \widehat{\theta}_{i, j}} \widehat{\theta}_{i, j} .
\end{aligned}
$$

Therefore, $M_{i, n_{i}-1}\left(Z_{i, n_{i}}\right)$ can be defined as

$$
M_{i, n_{i}-1}\left(Z_{i, n_{i}}\right)=-\sum_{j=1}^{n_{i}-1} \frac{z_{i, n_{i}}^{2}\left(\partial \nu_{i, n_{i}-1} / \partial \widehat{\theta}_{i, j}\right)^{2}\left(\left(\gamma_{i} / 2 \eta_{i, j}^{2}\right) S^{2} z_{i, j}^{2}\right)^{2}}{\sqrt{z_{i, k}^{2}\left(\partial \nu_{i, n_{i}-1} / \partial \hat{\theta}_{i, j}\right)^{2}\left(\left(\gamma_{i} / 2 \eta_{i, j}^{2}\right) S^{2} z_{i, j}^{2}\right)^{2}+\kappa^{2}}}-\lambda_{i} z_{i, n_{i}} \sum_{j=1}^{n_{i}-1} \frac{\partial v_{i, n_{i}-1}}{\partial \widehat{\theta}_{i, j}} \widehat{\theta}_{i, j}
$$

with the result that

Substituting (71) into(68) yields

$$
z_{i, n_{i}}\left(M_{i, n_{i}-1}\left(Z_{i, n_{i}}\right)-\sum_{j=1}^{n_{i}-1} \frac{\partial v_{i, n_{i}-1}}{\partial \widehat{\theta}_{i, j}} \dot{\theta}_{i, j}\right) \leq 0
$$

$$
\dot{V}_{i, n_{i}} \leq-2^{(1+\alpha) / 2} \sum_{j=1}^{n_{i}} \varsigma_{i, j 1}\left(\frac{1}{2} z_{i, j}^{2}+\frac{b_{i} \widetilde{\theta}_{i, j}^{2}}{2 \gamma_{i}}\right)^{(1+\alpha) / 2}-2 \sum_{j=1}^{n_{i}} \varsigma_{i, j 2}\left(\frac{1}{2} z_{i, j}^{2}+\frac{b_{i} \widetilde{\theta}_{i, j}^{2}}{2 \gamma_{i}}\right)^{(1+\beta) / 2}+\tau_{i},
$$

where $\tau_{i}=\sum_{j=1}^{n_{i}} C_{i, j}$.

Making $\quad \phi_{i, 1}=\min 2^{(1+\alpha) / 2}\left\{\varsigma_{i, 11}, \varsigma_{i, 21}, \ldots, \varsigma_{i, n_{i} 1}\right\}$ and $\phi_{i, 2}=\min 2\left\{\varsigma_{i, 12}, \varsigma_{i, 22}, \ldots, \varsigma_{i, n_{i}}\right\}$, in accordance with Lemma 4, yields

$$
\begin{aligned}
& -2^{(1+\alpha) / 2} \sum_{j=1}^{n_{i}} \varsigma_{i, j 1}\left(\frac{1}{2} z_{i, j}^{2}+\frac{b_{i} \widetilde{\theta}_{i, j}^{2}}{2 \gamma_{i}}\right)^{(1+\alpha) / 2} \leq-\phi_{i, 1}\left(\sum_{j=1}^{n_{i}}\left(\frac{1}{2} z_{i, j}^{2}+\frac{b_{i} \widetilde{\theta}_{i, j}^{2}}{2 \gamma_{i}}\right)\right)^{(1+\alpha) / 2}, \\
& -2 \sum_{j=1}^{n_{i}} \varsigma_{i, j 2}\left(\frac{1}{2} z_{i, j}^{2}+\frac{b_{i} \widetilde{\theta}_{i, j}^{2}}{2 \gamma_{i}}\right)^{(1+\beta) / 2} \leq-n_{i}^{(1+\beta) / 2} \phi_{i, 2}\left(\sum_{j=1}^{n_{i}}\left(\frac{1}{2} z_{i, j}^{2}+\frac{b_{i} \widetilde{\theta}_{i, j}^{2}}{2 \gamma_{i}}\right)\right)^{(1+\beta) / 2},
\end{aligned}
$$

and then substituting (73) and (74) into (72) yields

$$
\dot{V}_{i, n_{i}} \leq-\phi_{i, 1} V_{i, n_{i}}^{(1+\alpha) / 2}-n_{i}^{(1+\beta) / 2} \phi_{i, 2} V_{i, n_{i}}^{(1+\beta) / 2}+\tau_{i} .
$$

Up to this point, the design of the ith follower's controller is finished.

\section{Stability Analysis}

Theorem 1. Consider the multiple uncertain nonlinear strict-feedback system (15) controlled by the distributed fixedtime consensus controller (65) and assume that the leader has 
directed paths to all followers 1 to $M$. Under Assumptions 1-3, virtual controllers (27), (41), (53), and (65), and the adaptive laws (33), (42), (54), and (66), the consensus tracking errors between the leader and the followers in the overall closed-loop system are CSUUB and can be made arbitrarily small within fixed-time, and the upper limit of the fixed convergence time is irrelevant to the initial state.

Proof. For the stability analysis, choose the total Lyapunov candidate function $V$ as

$$
V=\sum_{i=1}^{M} V_{i, n_{i}}
$$

Differentiating (76) and substituting (75) into it, we have

$$
\dot{V} \leq-\sum_{i=1}^{M} \phi_{i, 1} V_{i, n_{i}}^{(1+\alpha) / 2}-\sum_{i=1}^{M} n_{i}^{(1+\beta) / 2} \phi_{i, 2} V_{i, n_{i}}^{(1+\beta) / 2}+\sum_{i=1}^{M} \tau_{i} .
$$

Making $\rho_{1}=\min \left\{\varphi_{1,1}, \varphi_{2,1}, \ldots, \varphi_{M, 1}\right\} \tau=\sum_{i=1}^{M} \tau_{i} \quad$ and $\rho_{2}=\min \left\{n_{1}^{(1+\beta) / 2} M^{(1+\beta) / 2} \varphi_{1,2}, \ldots, n_{M}^{(1+\beta) / 2} M^{(1+\beta) / 2} \phi_{M, 2}\right\}$, in accordance with Lemma 4 yields

$$
\begin{array}{r}
-\sum_{i=1}^{M} \phi_{i, 1} V_{i, n_{i}}^{(1+\alpha) / 2} \leq-\rho_{1}\left(\sum_{i=1}^{M} V_{i, n_{i}}\right)^{(1+\alpha) / 2}, \\
-\sum_{i=1}^{M} n_{i}^{(1+\beta) / 2} \phi_{i, 2} V_{i, n_{i}}^{(1+\beta) / 2} \leq-\rho_{2}\left(\sum_{i=1}^{M} V_{i, n_{i}}\right)^{(1+\beta) / 2},
\end{array}
$$

where $\rho_{1}>0, \rho_{2}>0, \alpha \in(0,1]$, and $\beta \in(1, \infty)$.

Then, (77) can be rewritten as

$$
\dot{V} \leq-\rho_{1} V^{(1+\alpha) / 2}-\rho_{2} V^{(1+\alpha) / 2}+\tau .
$$

In accordance with Lemma 3, design proper parameters to make (79) satisfy the following situation.

Case 1. If $V>\left(\tau /\left((1-\varpi) \rho_{1}\right)\right)^{(1+\alpha) / 2}, \varpi \in(0,1)$, then (79) can be written as

$$
\dot{V} \leq-\omega \rho_{1} V^{(1+\alpha) / 2}-\rho_{2} V^{(1+\beta) / 2},
$$

and in that manner, the solution of system (15) converges on the compact set

$$
x \in\left\{V(x) \leq\left(\frac{\tau}{(1-\omega) \rho_{1}}\right)^{2 /(1+\alpha)}\right\} .
$$

The fixed convergence time is

$$
T \leq T_{\max }:=\frac{2}{\omega \rho_{1}(1-\alpha)}+\frac{2}{\rho_{2}(\beta-1)} .
$$

Case 2. If $V>\left(\tau /\left((1-\Phi) \rho_{2}\right)\right)^{(1+\beta) / 2}, \varpi \in(0,1)$, then (79) can be written as

$$
\dot{V} \leq-\rho_{1} V^{(1+\alpha) / 2}-\omega \rho_{2} V^{(1+\beta) / 2},
$$

and in that manner, the solution of system (16) converges on the compact set

$$
x \in\left\{V(x) \leq\left(\frac{\tau}{(1-\Phi) \rho_{2}}\right)^{2 /(1+\beta)}\right\} .
$$

The fixed convergence time is

$$
T \leq T_{\max }:=\frac{2}{\rho_{1}(1-\alpha)}+\frac{2}{\omega \rho_{2}(\beta-1)} .
$$

When cases 1 and 2 are combined, the solution of system (15) converges on

$$
x \in\left\{V(x) \leq \min \left\{\left(\frac{\tau}{(1-\Phi) \rho_{1}}\right)^{2 /(1+\alpha)},\left(\frac{\tau}{(1-\Phi) \rho_{2}}\right)^{2 /(1+\beta)}\right\}\right\} .
$$

The fixed convergence time is

$$
T_{s} \leq T_{\max }:=\frac{2}{\omega \rho_{1}(1-\alpha)}+\frac{2}{\omega \rho_{2}(\beta-1)} .
$$

It can be seen from (80) and (83) that $V$ is bounded, with the result that $z_{i, j}$ and $\widetilde{\theta}_{i, j}$ are bounded, where $i=1, \ldots, M$ and $j=1, \ldots, n_{i}$, as $\widehat{\theta}_{i, j}=\theta_{i, j}-\widetilde{\theta}_{i, j}$ is also bounded. Because $z_{i, 1}=\sum_{j=1}^{M} a_{i j}\left(y_{i}-y_{j}\right)+h_{i}\left(y_{i}-y_{0}\right)$ and $z_{i, 1}$ and $y_{0}$ are bounded, $y_{i}$ are bounded. Because $z_{i, 2}=x_{i, 2}-v_{i, 1}, x_{i, 2}$ are bounded. Similarly, $v_{i, k-1}$ and $x_{i, k}, k=1, \ldots, n_{i}$, are bounded. Therefore, all signals in the overall closed-loop system are CSUUB.

Remark 11. The distributed fixed-time consensus control algorithm in this paper is different from previous control algorithms. The principles are as follows. (1) Compared to the fixed-time control algorithm proposed in [16-26], our control strategy is applicable to higher-order agents with various forms. That is, a group of the followers with the different unknown nonlinear functions and order of the dynamics can be considered in this brief. (2) To solve the problem of "explosion of complexity", instead of using dynamic surface design technique [33], we construct a smooth function $M(Z)$, from Lyapunov stability theorem, to compensate the part of Virtual Controller's derivative $\left(\partial v_{i} / \partial \hat{\theta}_{i}\right) \widehat{\theta}_{i}$ and using RBFNNs to approximate the rest of it. (3) $\mathrm{RBF}$ neural networks are used here to approximate unknown complex nonlinear functions, obviating the designed control protocol just work for special systems.

\section{Simulation Results}

In this section, we present two simulation results to validate the proposed theoretical result.

5.1. Mathematical Example. We consider a group of one leader and four followers with the following nonlinear strictfeedback dynamics in the $2 \mathrm{D}$ space (i.e., $p=2$ ) described by [33] 


$$
\begin{aligned}
\dot{x}_{i, 1} & =x_{i, 2}+f_{i, 1}\left(x_{i, 1}\right), \\
\dot{x}_{i, 2} & =u_{i}+f_{i, 2}\left(x_{i}\right), \\
y_{i} & =x_{i, 1},
\end{aligned}
$$

where $\quad i=1, \ldots, 4, \quad x_{i, k}=\left[x_{i, k 1}, x_{i, k 2}\right]^{T} ; \quad k=1,2$, $x_{i}=\left[x_{i, 1}^{T}, x_{i, 2}^{T}\right]^{T}, y_{i}=\left[y_{i, 1}, y_{i, 2}\right]^{T}, f_{i, 1}\left(x_{i, 1}\right)=\left[\sin \left(x_{i, 11}\right) x_{i .12}\right.$, $\left.\cos \left(x_{i, 12}\right)\right]^{T}$, and $f_{i, 2}\left(x_{i}\right)=e^{-0.4 x_{i, 1}} x_{i, 21}\left[\cos \left(x_{i, 21}\right), x_{i, 22}\right]^{T}$.

The directed network topology for the simulation is shown in Figure 1 . We choose $a_{i j}=1$ on $j \in N_{i}, a_{i j}=0$ otherwise, $h_{1}=h_{2}=h_{3}=0$, and $h_{4}=1$. The leader output $r(t)$ is $r(t)=\left[r_{1}(t), r_{2}(t)\right]=[4 \sin (t / 20), \cos (0.5 t)+0.5 \sin$ $(1.5 t)]^{T}$ and the initial states and adaptive laws of the four followers are set to $x_{1}(0)=[1,0,1,-1]^{T}, x_{2}(0)=[-1,-2,1$, $-3]^{T}, x_{3}(0)=[0,-2,2,-2]^{T}$, and $x_{4}(0)=[-1,-1,2,3]^{T}$ and $\widehat{\theta}_{1}(0)=[0,0,0,0]^{T}, \widehat{\theta}_{2}(0)=[0,0,0,0]^{T}, \widehat{\theta}_{3}(0)=[0,0,0,0]^{T}$, and $\widehat{\theta}_{4}(0)=[0,0,0,0]^{T}$, respectively. To display the consensus tracking errors between the leader and the followers, we use $e_{i}=\left\|x_{i, 1}(t)-r(t)\right\|$, where $i=1, \ldots, 4$. And the design parameters are chosen as follows: $\alpha=1 / 3, \beta=5$, $\varsigma_{i, 11}=\varsigma_{i, 12}=\varsigma_{i, 21}=\varsigma_{i, 22}=5, \varsigma_{i, 13}=\varsigma_{i, 23}=0.1, b_{i}=5, \gamma_{i}=5$, $\eta_{i, 1}=\eta_{i, 2}=0.25, \quad \varepsilon_{10}=\varepsilon_{20}=0.001$, and $\lambda_{i}=0.04$, where $i=1, \ldots, 4$. It is worth noting that $\varsigma_{i, 11}=\varsigma_{i, 12}=\varsigma_{i, 21}=\varsigma_{i, 22}$ should be at least ten times that of $\varsigma_{i, 13}=\varsigma_{i, 23}$ and $b_{i}$ setting of 5 is much better than setting it to 2 . For the simulation, RBF neural networks are used to estimate unknown nonlinear functions, where $W_{i, 1} S_{i, 1}\left(Z_{i, 1}\right)$ and $W_{i, 2} S_{i, 2}\left(Z_{i, 2}\right)$ contain nine nodes, and the center of the Gaussian function are set as

$$
\xi_{i, 1}=\left[\begin{array}{lllllllll}
-2 & -1.5 & -1 & -0.5 & 0 & 0.5 & 1 & 1.5 & 2 \\
-2 & -1.5 & -1 & -0.5 & 0 & 0.5 & 1 & 1.5 & 2 \\
-2 & -1.5 & -1 & -0.5 & 0 & 0.5 & 1 & 1.5 & 2 \\
-2 & -1.5 & -1 & -0.5 & 0 & 0.5 & 1 & 1.5 & 2 \\
-2 & -1.5 & -1 & -0.5 & 0 & 0.5 & 1 & 1.5 & 2 \\
-2 & -1.5 & -1 & -0.5 & 0 & 0.5 & 1 & 1.5 & 2 \\
-2 & -1.5 & -1 & -0.5 & 0 & 0.5 & 1 & 1.5 & 2 \\
-2 & -1.5 & -1 & -0.5 & 0 & 0.5 & 1 & 1.5 & 2
\end{array}\right],
$$

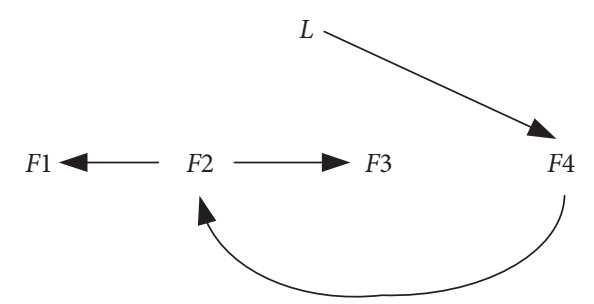

FIGURE 1: Network topology for a group of one leader (L) and four followers F1 to F4.

where $i=1, \ldots, 4$. Figure 2 shows the outputs of the four followers and one leader in the 2D space. Figure 3 shows the consensus tracking errors $e_{i}$ and we can find it drop quickly in less than 0.3 second. Figure 4 shows the state $x_{i, 1}$ and $x_{i, 2}$ of the four followers. Figure 5 shows the adaptive laws $\widehat{\theta}_{i}$. These figures reveal that the consensus tracking between the leader and followers is achieved satisfactorily under a directed network topology, although the followers have the unknown nonlinearities unmatched in the control input.

5.2. Practical Example. Consider multiple one-link robotic manipulators consisting of four followers and a leader. The dynamic of each follower manipulator is described as

$$
\left\{M \ddot{q}(t)+\frac{1}{2} m g l \sin q(t)=u(t), y(t)=q(t),\right.
$$

where $q(t)$ represents angular position; $M$ denotes the instantaneous inertia; $u(t)$ is the input torque; $g$ is the gravitational torque; and $m$ and $l$ are the mass and the length of the rod, respectively.

Perform coordinate transformation on system (90) and set $x_{i, 1}(t)=q(t)$ and $x_{i, 2}(t)=\dot{q}(t)$; then, system (90) can be rewritten as follows:

$$
\left\{\begin{array}{l}
\dot{x}_{i, 1}(t)=x_{i, 2}(t), \\
\dot{x}_{i, 2}(t)=\frac{1}{M}\left(u(t)-\frac{1}{2} m g l \sin x_{i, 1}(t)\right), \\
y_{i}(t)=x_{i, 1}(t)
\end{array}\right.
$$

where $i=1, \ldots, 4$. The directed network topology for this practical simulation is also shown in Figure 1. We choose $a_{i j}=1$ on $j \in N_{i}, a_{i j}=0$ otherwise, $h_{1}=h_{2}=h_{3}=$ 0 and $h_{4}=1$. The leader output $r(t)$ is $y_{0}(t)=0.25 \sin (t)$ and the initial states and adaptive laws of the four followers are set to $x_{1}(0)=[0.1,-0.2]^{T}, x_{2}(0)=[0.15,-0.1]^{T}$, $x_{3}(0)=[0.2,0.2]^{T}$, and $x_{4}(0)=[0.12,-0.2]^{T}$ and $\widehat{\theta}_{1}(0)=$ $[0,0]^{T}, \widehat{\theta}_{2}(0)=[0,0]^{T}, \widehat{\theta}_{3}(0)=[0,0]^{T}$, and $\widehat{\theta}_{4}(0)=[0,0]^{T}$, respectively. To display the consensus tracking errors between the leader and the followers, we use $e_{i}=$ $x_{i, 1}(t)-r(t)$, where $i=1, \ldots, 4$. And the design parameters are chosen as follows: $M=1, m=1, l=1, g=$ $9.8 \alpha=1 / 3, \beta=5, \varsigma_{i, 11}=\varsigma_{i, 12}=\varsigma_{i, 21}=\varsigma_{i, 22}=5, \varsigma_{i, 13}=\varsigma_{i, 23}=$ $0.1, b_{i}=5, \gamma_{i}=5, \eta_{i, 1}=\eta_{i, 2}=0.25, \varepsilon_{10}=\varepsilon_{20}=0.001$, and $\lambda_{i}=0.04$, where $i=1, \ldots, 4$. For the simulation, RBF neural networks are used to estimate unknown nonlinear 


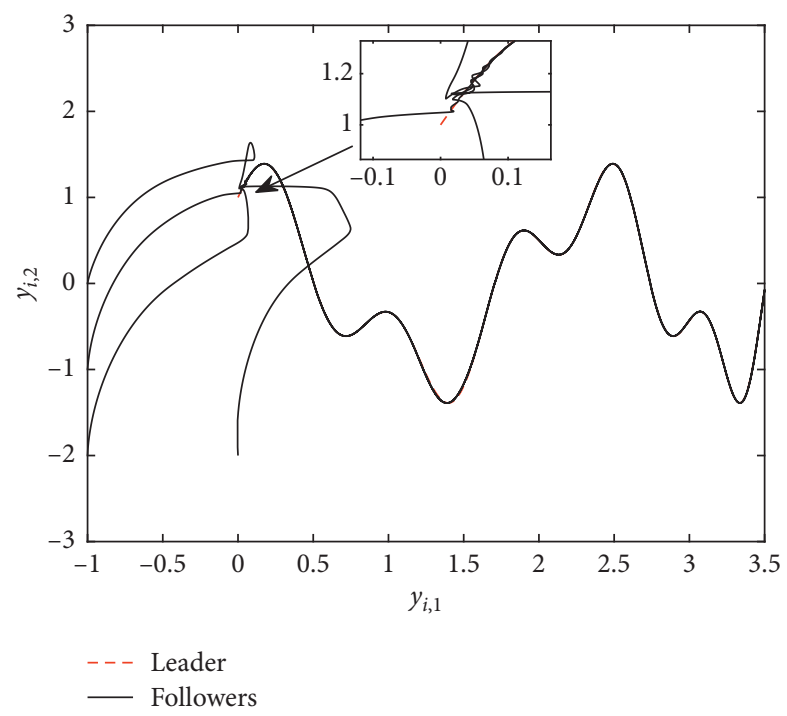

FIgURE 2: Output trajectories of the followers with one leader in the $2 \mathrm{D}$ space.

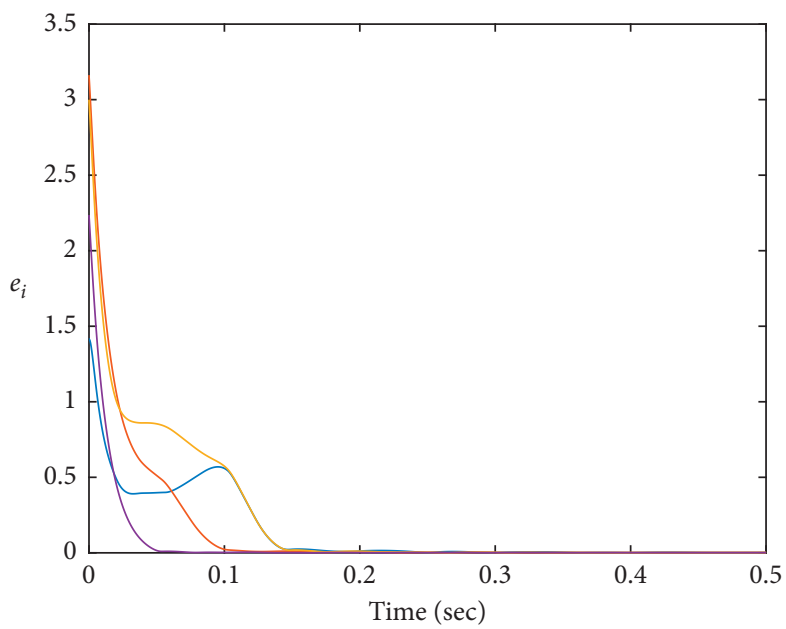

FIgURE 3: The tracking errors $e_{i}$ between followers and leader.

functions, where $W_{i, 1} S_{i, 1}\left(Z_{i, 1}\right)$ and $W_{i, 2} S_{i, 2}\left(Z_{i, 2}\right)$ contain nine nodes, and the center of the Gaussian function are set as

$$
\begin{aligned}
\xi_{i, 1} & =\left[\begin{array}{lllllllll}
-2 & -1.5 & -1 & -0.5 & 0 & 0.5 & 1 & 1.5 & 2 \\
-2 & -1.5 & -1 & -0.5 & 0 & 0.5 & 1 & 1.5 & 2 \\
-2 & -1.5 & -1 & -0.5 & 0 & 0.5 & 1 & 1.5 & 2 \\
-2 & -1.5 & -1 & -0.5 & 0 & 0.5 & 1 & 1.5 & 2
\end{array}\right], \\
\xi_{i, 2} & =\left[\begin{array}{lllllllll}
-2 & -1.5 & -1 & -0.5 & 0 & 0.5 & 1 & 1.5 & 2 \\
-2 & -1.5 & -1 & -0.5 & 0 & 0.5 & 1 & 1.5 & 2 \\
-2 & -1.5 & -1 & -0.5 & 0 & 0.5 & 1 & 1.5 & 2 \\
-2 & -1.5 & -1 & -0.5 & 0 & 0.5 & 1 & 1.5 & 2 \\
-2 & -1.5 & -1 & -0.5 & 0 & 0.5 & 1 & 1.5 & 2 \\
-2 & -1.5 & -1 & -0.5 & 0 & 0.5 & 1 & 1.5 & 2 \\
-2 & -1.5 & -1 & -0.5 & 0 & 0.5 & 1 & 1.5 & 2
\end{array}\right],
\end{aligned}
$$

where $i=1, \ldots, 4$. Then, by using the scheme in [36], simulation results are given in Figures 6(a) and 7(a). Meanwhile, by employing our method, simulation results are given in Figures 6(b), 7(b), 8, and 9.

It is shown in Figures 6 and 7 that the tracking performance by using our method is better than [36], since our proposed scheme has faster convergence and higher precision. Figure 8 shows the states $x_{i, 2}$ of the four followers. Figure 9 shows the response curves of adaptive laws $\widehat{\theta}_{i, 2}$. These figures reveal that the consensus tracking between the leader and followers is achieved satisfactorily under a directed network topology.

\section{Conclusion}

In this paper, the distributed adaptive fixed-time tracking consensus control problem is investigated for multiple strictfeedback systems with uncertain nonlinearities under a 

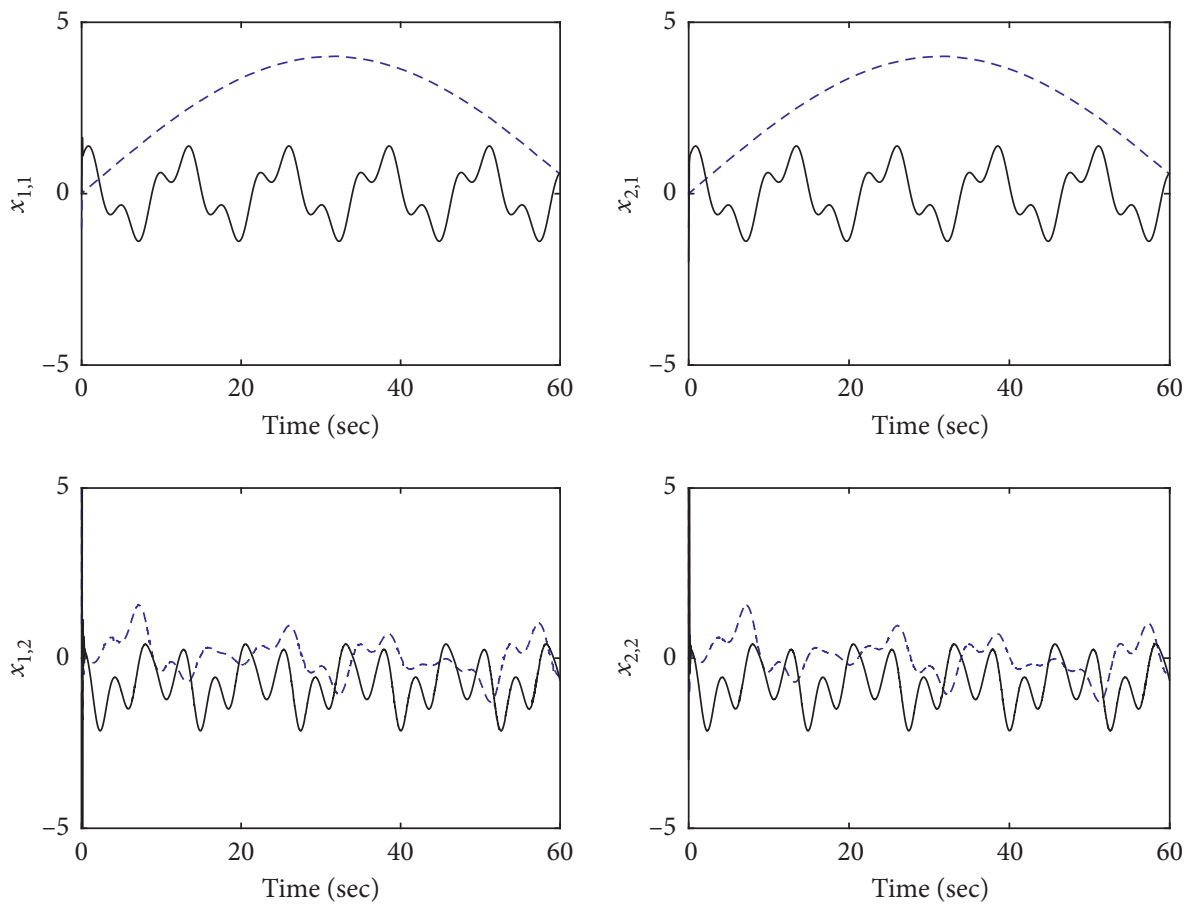

(a)

(b)
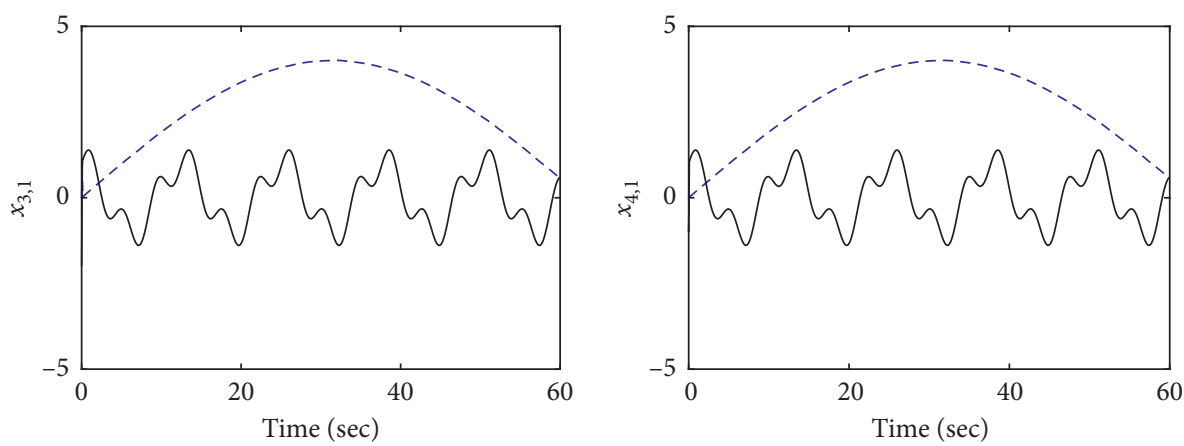

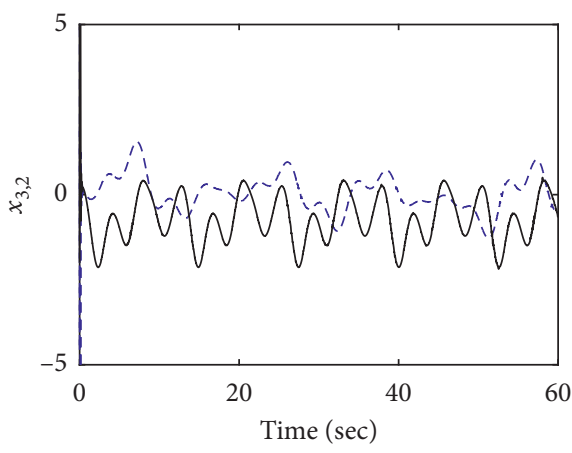

(c)

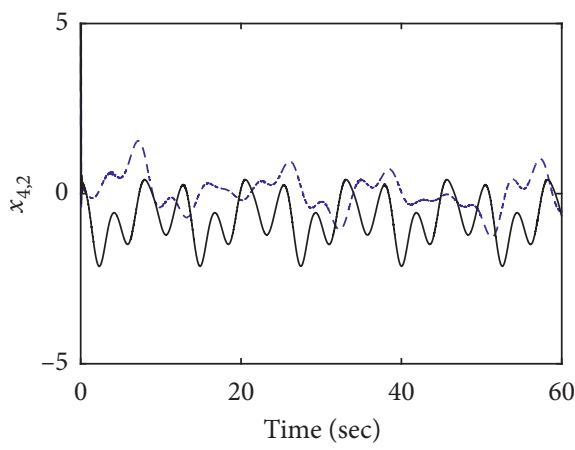

(d)

Figure 4: The states of the four followers. (a) $x_{1,1}$ and $x_{1,2}$. (b) $x_{2,1}$ and $x_{2,2}$. (c) $x_{3,1}$ and $x_{3,2}$. (d) $x_{4,1}$ and $x_{4,2}$. 

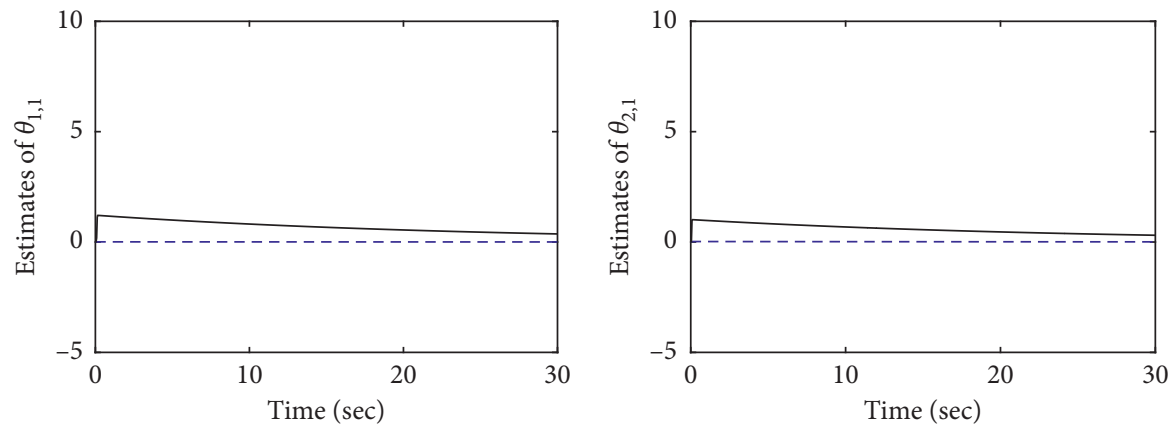

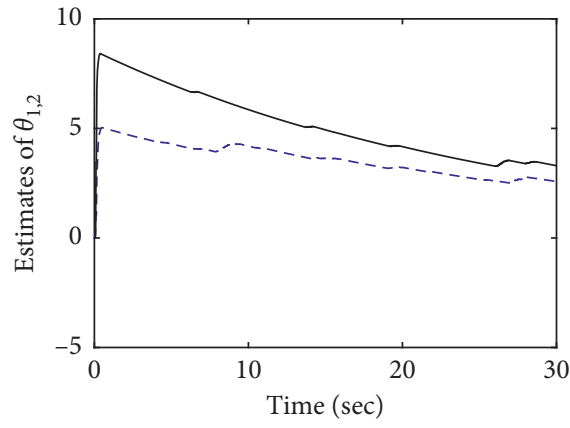

(a)
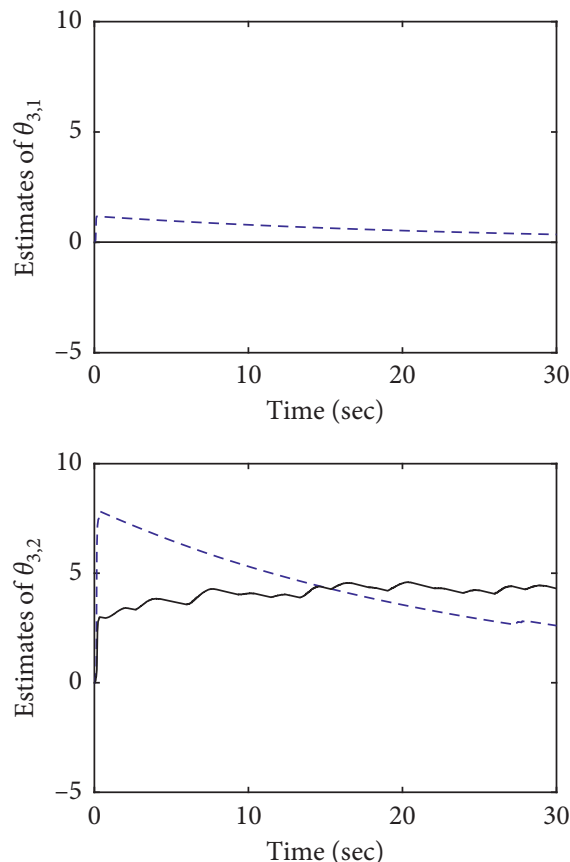

(c)

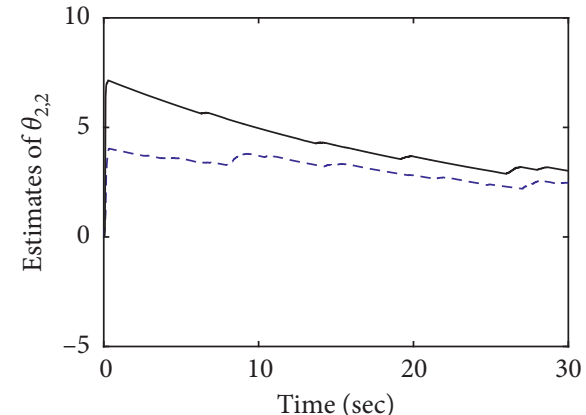

(b)
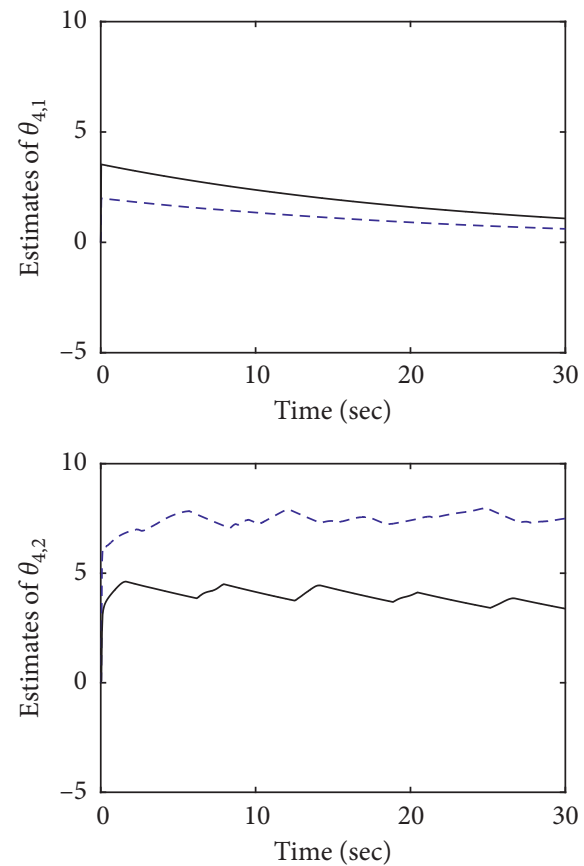

(d)

FIgure 5: The adaptive laws of the four followers. (a) $\widehat{\theta}_{1,1}$ and $\widehat{\theta}_{1,2}$ (b) $\widehat{\theta}_{2,1}$ and $\widehat{\theta}_{2,2}$. (c) $\widehat{\theta}_{3,1}$ and $\widehat{\theta}_{3,2}$. (d) $\widehat{\theta}_{4,1}$ and $\widehat{\theta}_{4,2}$. 


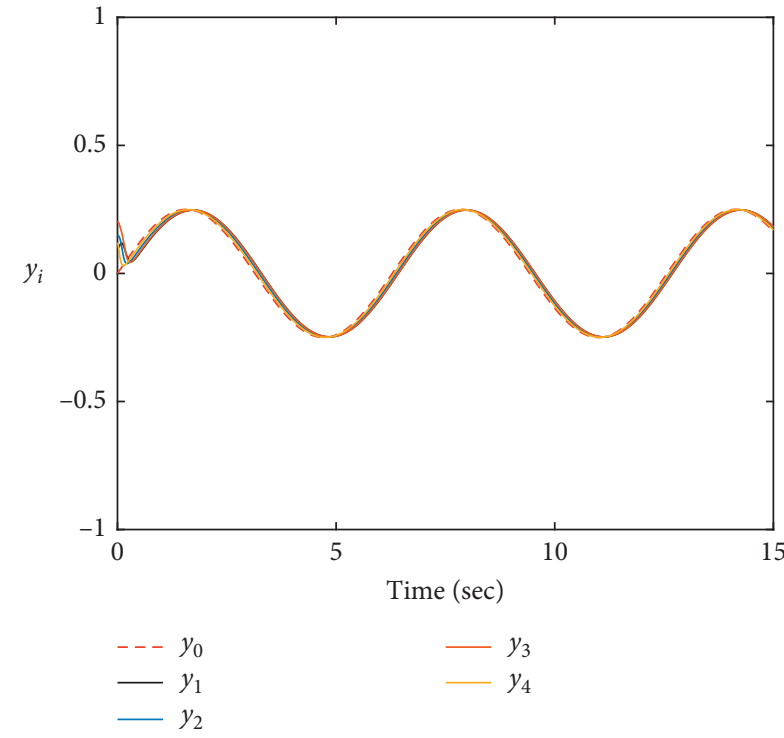

(a)

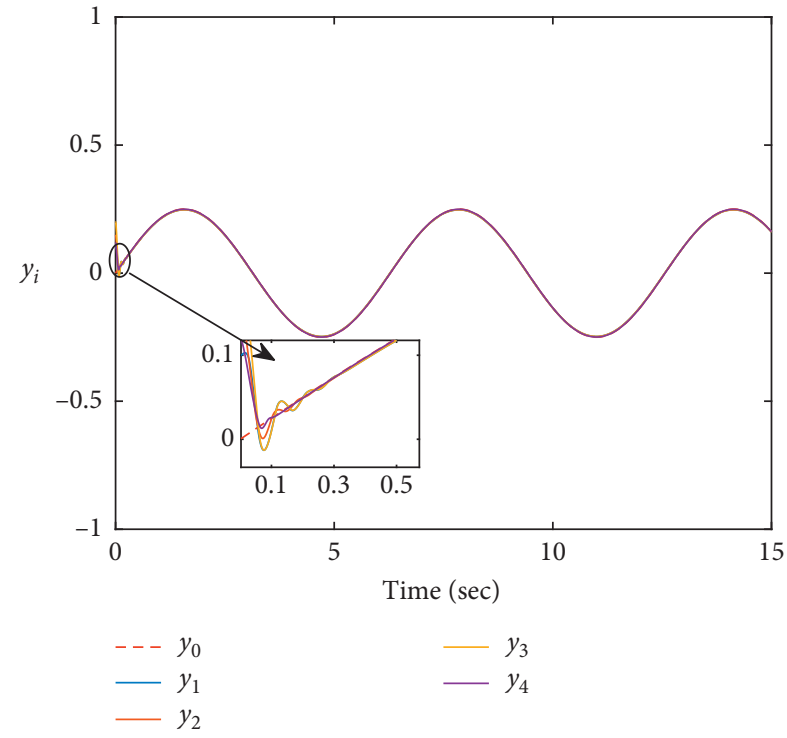

(b)

FIgURE 6: Output trajectories of the followers with one leader by using two methods. (a) Output trajectories by the method in [36]. (b) Output trajectories by our method.

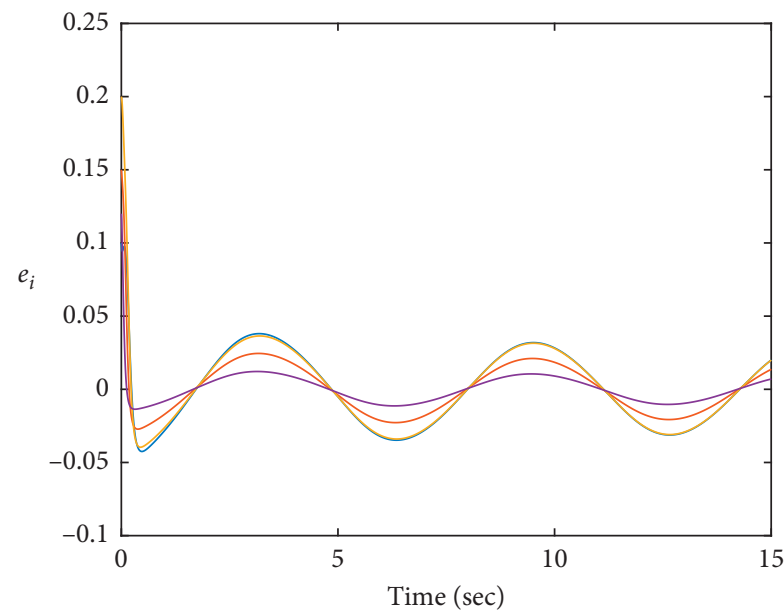

(a)

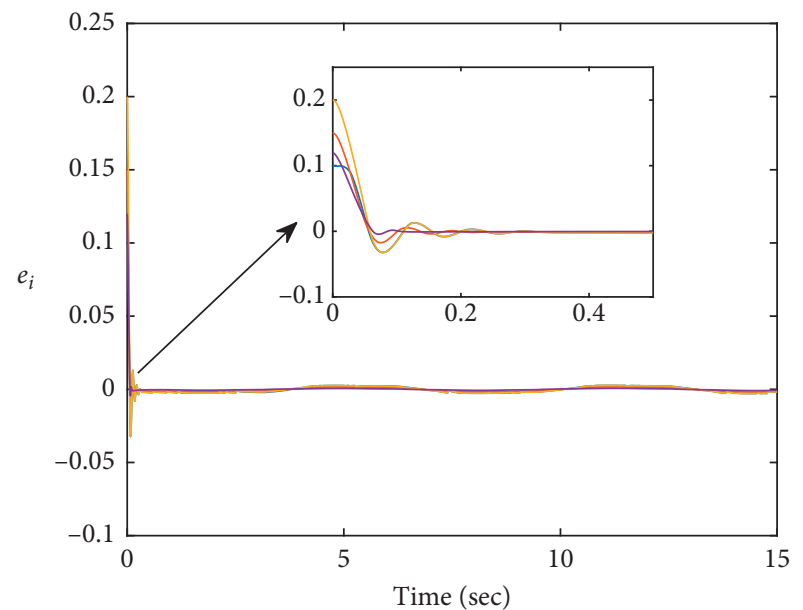

(b)

FIgURE 7: The tracking errors $e_{i}$ between followers and leader by using two methods. (a) Error curves by the method in [36]. (b) Error curves by our method.

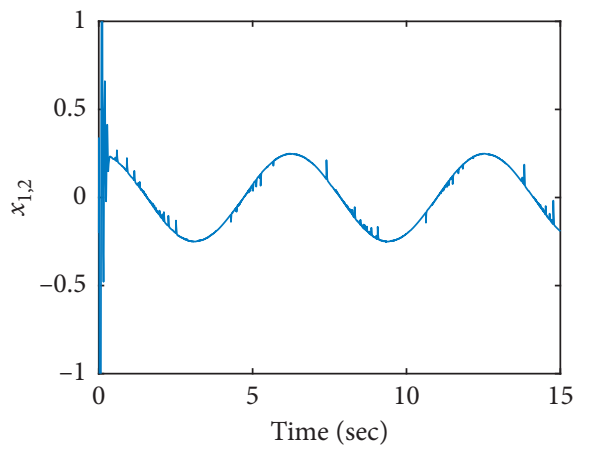

(a)

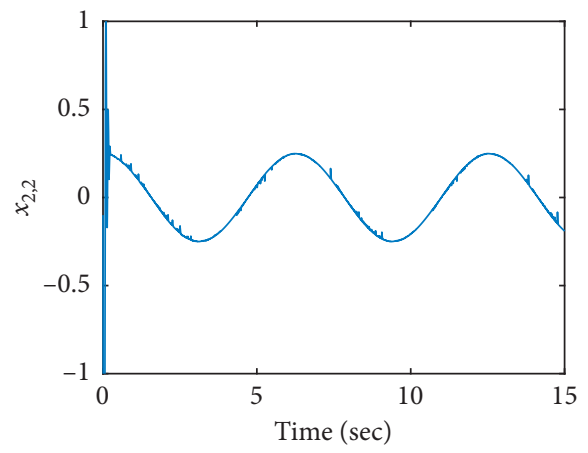

(b)

Figure 8: Continued. 


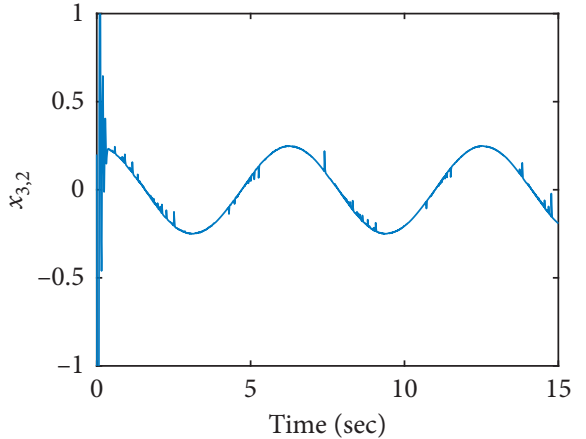

(c)

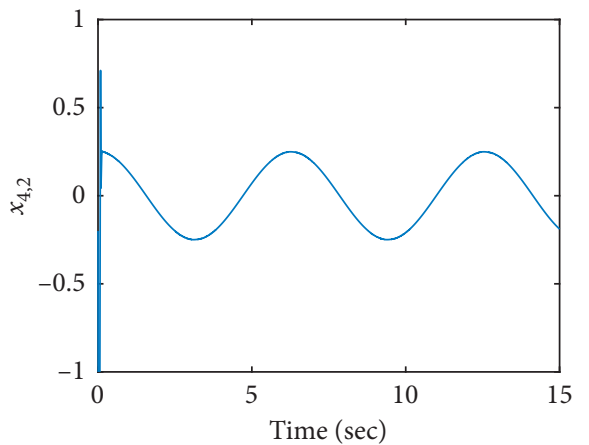

(d)

Figure 8: The states $x_{i, 2}$ of the four followers.

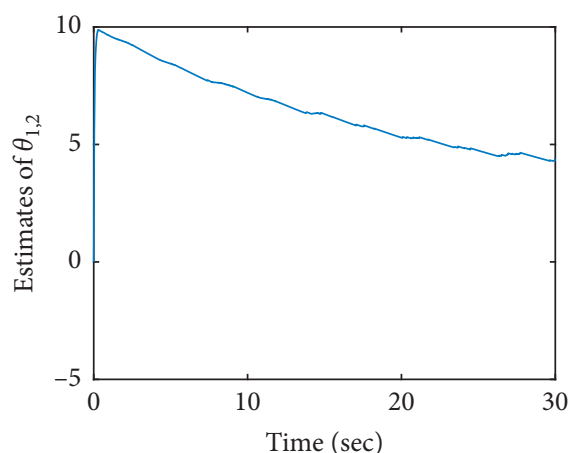

(a)

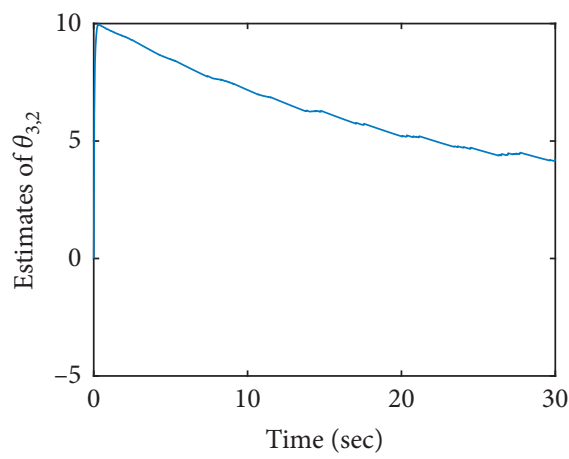

(c)

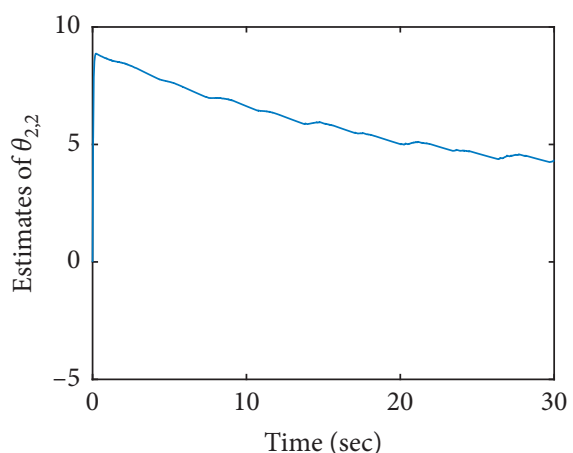

(b)

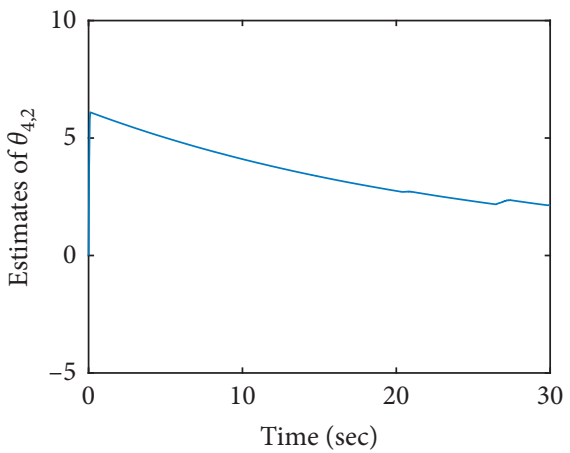

(d)

FIgURE 9: The adaptive laws $\hat{\theta}_{i, 2}$ of the four followers.

directed graph topology. The function approximation technique using RBF neural networks was employed to compensate for the unknown nonlinearities unmatched in the control input of followers. From Lyapunov stability and fixed-time control theory, by using distributed coordinate conversion, designed virtual controllers, and actual controllers. Based on Lyapunov stability theory, it is proved that the developed distributed consensus protocol can guarantee all the signals in multiple strict-feedback systems are bounded, and the tracking consensus errors converge to a small neighborhood of zero in fixed time. Finally, the effectiveness of the proposed approach was proved through two simulations. And in the following research, we will consider the application of fixed-time control in pure-feedback nonlinear systems with time delay.

\section{Data Availability}

No data were used to support this study.

\section{Conflicts of Interest}

The authors declare that they have no conflicts of interest. 


\section{Acknowledgments}

This work was supported in part by the National Natural Science Foundation of China under Grants 61663032 and 61663030.

\section{References}

[1] A. Polyakov, "Nonlinear feedback design for fixed-time stabilization of linear control systems," IEEE Transactions on Automatic Control, vol. 57, no. 8, pp. 2106-2110, 2011.

[2] X. Lu, Y. Wang, X. Yu et al., "Finite-time control for robust tracking consensus in MASs with an uncertain leader," IEEE Transactions on Cybernetics, vol. 47, no. 5, pp. 1210-1223, 2016.

[3] X. Wang, S. Li, and P. Shi, "Distributed finite-time containment control for double-integrator multiagent systems," IEEE Transactions on Cybernetics, vol. 44, no. 9, pp. 15181528, 2014.

[4] S. Yu and X. Long, "Finite-time consensus for second-order multi-agent systems with disturbances by integral sliding mode," Automatica, vol. 54, pp. 158-165, 2015.

[5] H. Du, G. Wen, G. Chen, J. Cao, and F. E. Alsaadi, "A distributed finite-time consensus algorithm for higher-order leaderless and leader-following multiagent systems," IEEE Transactions on Systems, Man, and Cybernetics: Systems, vol. 47, no. 7, pp. 1625-1634, 2017.

[6] X. Liu, J. Cao, N. Jiang, G. Hao, and S. Wang, "Finite-time consensus of second-order multi-agent systems via auxiliary system approach," Journal of the Franklin Institute, vol. 353, no. 7, pp. 1479-1493, 2016.

[7] H. Yu, Y. Shen, and X. Xia, "Adaptive finite-time consensus in multi-agent networks," Systems \& Control Letters, vol. 62, no. 10, pp. 880-889, 2013.

[8] L. Wang and F. Xiao, "Finite-time consensus problems for networks of dynamic agents," IEEE Transactions on Automatic Control, vol. 55, no. 4, pp. 950-955, 2010.

[9] H. Du, S. Li, and C. Qian, "Finite-time attitude tracking control of spacecraft with application to attitude synchronization," IEEE Transactions on Automatic Control, vol. 56, no. 11, pp. 2711-2717, 2011.

[10] S. P. Bhat and D. S. Bernstein, "Geometric homogeneity with applications to finite-time stability," Mathematics of Control, Signals, and Systems, vol. 17, no. 2, pp. 101-127, 2005.

[11] S. Li, H. Du, and X. Lin, "Finite-time consensus algorithm for multi-agent systems with double-integrator dynamics," Automatica, vol. 47, no. 8, pp. 1706-1712, 2011.

[12] Y. Zheng and L. Wang, "Finite-time consensus of heterogeneous multi-agent systems with and without velocity measurements," Systems \& Control Letters, vol. 61, no. 8, pp. 871-878, 2012.

[13] J. Huang, C. Wen, W. Wang, and Y.-D. Song, "Adaptive finite-time consensus control of a group of uncertain nonlinear mechanical systems," Automatica, vol. 51, pp. 292-301, 2015.

[14] Y. Wang, Y. Song, M. Krstic, and C. Wen, "Adaptive finite time coordinated consensus for high-order multi-agent systems: adjustable fraction power feedback approach," Information Sciences, vol. 372, pp. 392-406, 2016.

[15] Y. Zhou, X. Yu, C. Sun, and W. Yu, "Higher order finite-time consensus protocol for heterogeneous multi-agent systems," International Journal of Control, vol. 88, no. 2, pp. 285-294, 2015.

[16] S. E. Parsegov, A. E. Polyakov, and P. S. Shcherbakov, "Fixedtime consensus algorithm for multi-agent systems with integrator dynamics," IFAC Proceedings Volumes, vol. 46, no. 27, pp. 110-115, 2013.

[17] H. Hong, W. Yu, G. Wen et al., "Distributed robust fixed-time consensus for nonlinear and disturbed multiagent systems," IEEE Transactions on Systems, Man, and Cybernetics: Systems, vol. 47, no. 7, pp. 1464-1473, 2016.

[18] J. Fu and J. Wang, "Fixed-time coordinated tracking for second-order multi-agent systems with bounded input uncertainties," Systems \& Control Letters, vol. 93, pp. 1-12, 2016.

[19] J. Ni, L. Liu, C. Liu, and J. Liu, "Fixed-time leader-following consensus for second-order multiagent systems with input delay," IEEE Transactions on Industrial Electronics, vol. 64, no. 11, pp. 8635-8646, 2017.

[20] H. Li, M. Zhu, Z. Chu, H. Du, G. Wen, and N. D. Alotaibi, "Fixed-time synchronization of a class of second-order nonlinear leader-following multi-agent systems," Asian Journal of Control, vol. 20, no. 1, pp. 39-48, 2018.

[21] Z. Y. Zuo, "Leader-follower fixed-time consensus of multiagent systems with high-order integrator dynamics," International Journal of Control, vol. 90, no. 7, pp. 1420-1427, 2017.

[22] Z. Zuo, B. Tian, M. Defoort, and Z. Ding, "Fixed-time consensus tracking for multiagent systems with high-order integrator dynamics," IEEE Transactions on Automatic Control, vol. 63, no. 2, pp. 563-570, 2018.

[23] H. Yang and D. Ye, "Adaptive fixed-time bipartite tracking consensus control for unknown nonlinear multi-agent systems: an information classification mechanism," Information Sciences, vol. 459, pp. 238-254, 2018.

[24] H. Yang and D. Ye, "Distributed fixed-time consensus tracking control of uncertain nonlinear multiagent systems: a prioritized strategy," IEEE transactions on cybernetics, vol. 50, no. 6, pp. 2627-2638, 2019.

[25] H. Wang, W. Yu, G. Wen, and G. Chen, "Fixed-time consensus of nonlinear multi-agent systems with general directed topologies," IEEE Transactions on Circuits and Systems II: Express Briefs, vol. 66, no. 9, pp. 1587-1591, 2019.

[26] Y. Wu, Z. Wang, and Z. Huang, "Distributed fault detection for nonlinear multi-agent systems under fixed-time observer," Journal of the Franklin Institute, vol. 356, no. 13, pp. 7515-7532, 2019.

[27] I. Kanellakopoulos, P. V. Kokotovic, and A. S. Morse, "Systematic design of adaptive controllers for feedback linearizable systems," IEEE Transactions on Automatic Control, vol. 36, no. 11, pp. 1241-1253, 1991.

[28] M. Krstic, I. Kanellakopoulos, and P. Kokotovic, Systematic Design of Adaptive Controllers for Feedback Linearizable Systems, Wiley-Interscience, New York, NY, USA, 1995.

[29] M. M. Polycarpou and M. J. Mears, "Stable adaptive tracking of uncertain systems using nonlinearly parametrized on-line approximators," International Journal of Control, vol. 70, no. 3, pp. 363-384, 1998.

[30] M. M. Polycarpou, "Stable adaptive neural control scheme for nonlinear systems," IEEE Transactions on Automatic Control, vol. 41, no. 3, pp. 447-451, 1996.

[31] D. Swaroop, J. K. Hedrick, P. P. Yip, and J. C. Gerdes, "Dynamic surface control for a class of nonlinear systems," IEEE Transactions on Automatic Control, vol. 45, no. 10, pp. 1893-1899, 2000.

[32] D. Wang and J. Huang, "Neural network-based adaptive dynamic surface control for a class of uncertain nonlinear systems in strict-feedback form," IEEE Transactions on Neural Networks, vol. 16, no. 1, pp. 195-202, 2005.

[33] S. J. Yoo, "Distributed consensus tracking for multiple uncertain nonlinear strict-feedback systems under a directed 
graph," IEEE Transactions on Neural Networks and Learning Systems, vol. 24, no. 4, pp. 666-672, 2013.

[34] J. Huang, Y.-D. Song, W. Wang, C. Wen, and G. Li, "Smooth control design for adaptive leader-following consensus control of a class of high-order nonlinear systems with timevarying reference," Automatica, vol. 83, pp. 361-367, 2017.

[35] J. Huang, W. Wang, C. Wen, J. Zhou, and G. Li, "Distributed adaptive leader-follower and leaderless consensus control of a class of strict-feedback nonlinear systems: a unified approach," Automatica, vol. 118, p. 109021, 2020.

[36] D. Ba, Y.-X. Li, and S. Tong, "Fixed-time adaptive neural tracking control for a class of uncertain nonstrict nonlinear systems," Neurocomputing, vol. 363, pp. 273-280, 2019.

[37] Y. Sun, B. Chen, C. Lin, and H. Wang, "Finite-time adaptive control for a class of nonlinear systems with nonstrict feedback structure," IEEE Transactions on Cybernetics, vol. 48, no. 10, pp. 2774-2782, 2018.

[38] F. Wang, X. Zhang, B. Chen, C. Lin, X. Li, and J. Zhang, "Adaptive finite-time tracking control of switched nonlinear systems," Information Sciences, vol. 421, pp. 126-135, 2017.

[39] F. Wang, B. Chen, X. Liu, and C. Lin, "Finite-time adaptive fuzzy tracking control design for nonlinear systems," IEEE Transactions on Fuzzy Systems, vol. 26, no. 3, pp. 1207-1216, 2018.

[40] R. M. Sanner and J.-J. E. Slotine, "Gaussian networks for direct adaptive control," IEEE Transactions on Neural Networks, vol. 3, no. 6, pp. 837-863, 1992.

[41] A. J. Kurdila, F. J. Narcowich, and J. D. Ward, "Persistency of excitation in identification using radial basis function approximants," SIAM Journal on Control and Optimization, vol. 33, no. 2, pp. 625-642, 1995.

[42] C. Wang, D. J. Hill, S. S. Ge, and G. Chen, “An ISS-modular approach for adaptive neural control of pure-feedback systems," Automatica, vol. 42, no. 5, pp. 723-731, 2006.

[43] G. H. Hardy, J. E. Littlewood, and G. Polya, Inequalities, Cambridge University Press, Cambridge, UK, 1952.

[44] C. Wang and Y. Lin, "Decentralized adaptive tracking control for a class of interconnected nonlinear time-varying systems," Automatica, vol. 54, pp. 16-24, 2015.

[45] W. Ren, "On consensus algorithms for double-integrator dynamics," IEEE Transactions on Automatic Control, vol. 53, no. 6, pp. 1053-1059, 2008.

[46] J. Xu, "Adaptive fixed-time control for MIMO nonlinear systems with asymmetric output constraints using universal barrier functions," IEEE Transactions on Automatic Control, vol. 64, no. 7, pp. 3046-3053, 2018. 